\title{
A fractional notion of length and an associated nonlocal curvature*
}

\author{
Brian Seguin
}

\section{Contents}

1 Introduction 1

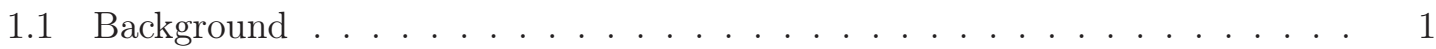

1.2 Extension and motivation . . . . . . . . . . . . . 3

2 Sets of discs

3 Fractional length $\quad 12$

4 Variation of $\operatorname{Len}_{\sigma}$ and nonlocal curvature 15

\begin{abstract}
Here a new notion of fractional length of a smooth curve, which depends on a parameter $\sigma$, is introduced that is analogous to the fractional perimeter functional of open sets. It is shown that in an appropriate limit the fractional length converges to the traditional notion of length up to a multiplicative constant. Since a curve that connects two points of minimal length must have zero curvature, the Euler-Lagrange equation associated with the fractional length is used to motivate a nonlocal notion of curvature for a curve. This is analogous to how the fractional perimeter has been used to define a nonlocal mean-curvature.

Dedicated to Eliot Fried, whose guidance following my time as a graduate student will always be appreciated.
\end{abstract}

\section{Introduction}

\subsection{Background}

The origins of fractional perimeter and nonlocal curvature began with the work of Caffarelli, Roquejoffre, and Savin [4] who defined, up to a multiplicative constant, the $\sigma$-perimeter,

\footnotetext{
*This is a corrected version of my paper [20] published under the same name.
} 
for $0<\sigma<1$, of a measurable set $E \subseteq \mathbb{R}^{n}$ relative to an open, bounded set $\Omega \subseteq \mathbb{R}^{n}$ by

$$
\operatorname{Per}_{\sigma}(E, \Omega):=\mathcal{I}\left(E \cap \Omega, E^{c} \cap \Omega\right)+\mathcal{I}\left(E \cap \Omega, E^{c} \cap \Omega^{c}\right)+\mathcal{I}\left(E \cap \Omega^{c}, E^{c} \cap \Omega\right),
$$

where

$$
\mathcal{I}(A, B):=\frac{1}{\alpha_{n-1}} \int_{A} \int_{B}|x-y|^{-n-\sigma} d x d y, \quad A \cap B=\emptyset,
$$

and $\alpha_{n-1}$ is the volume of the unit ball in $\mathbb{R}^{n-1}$. The first term in this definition is related to the fractional Sobolev space seminorm $\left|\chi_{E}\right|_{H^{\sigma / 2}(\Omega)}$, and can be viewed as the fractional perimeter of $E$ inside of $\Omega$, while the other two terms can be interpreted as the fractional perimeter near $\partial \Omega$. The study of functionals of this kind goes back to the work of Visintin [22. It is known [5] that if the boundary of $E$ is smooth, then

$$
\lim _{\sigma \uparrow 1}(1-\sigma) \operatorname{Per}_{\sigma}\left(E, B_{r}\right)=\mathcal{H}^{n-1}\left(\partial E \cap B_{r}\right)
$$

for almost every $r>0$, where $B_{r}$ is the ball centered at the origin of radius $r$. A set $E \subseteq \mathbb{R}^{n}$ is a minimizer of the $\sigma$-perimeter relative to $\Omega$ if over all measurable sets $F \subseteq \mathbb{R}^{n}$ such that $E \backslash \Omega=F \backslash \Omega$ we have

$$
\operatorname{Per}_{\sigma}(E, \Omega) \leq \operatorname{Per}_{\sigma}(F, \Omega)
$$

Besides the relation (3), it is known that the $\sigma$-perimeter functional $\Gamma$-converges to the classical notion of perimeter [3].

If the boundary of a minimizer $E$ is sufficiently regular, then it must satisfy

$$
\int_{\mathbb{R}^{n}} \frac{\tilde{\chi}_{E}(x)}{|z-x|^{n+\sigma}} d x=0 \quad \text { for all } z \in \partial E
$$

where $\tilde{\chi}_{E}:=\chi_{E}-\chi_{E^{c}}, \chi_{E}$ is the characteristic function for the set $E$, and this integral is taken in the principle-value sense. Because of the connection between the $\sigma$-perimeter and the areal measure (3), and the fact that surfaces that minimize their area subject to a fixed boundary condition must have zero mean curvature, it is reasonable to define a nonlocal mean-curvature by

$$
H_{\sigma}(z):=\frac{1}{\omega_{n-2}} \int_{\mathbb{R}^{n}} \frac{\tilde{\chi}_{E}(x)}{|z-x|^{n+\sigma}} d x \quad \text { for all } z \in \partial E,
$$

where $\omega_{n-2}$ is the $(n-2)$-dimensional measure of the unit sphere in $\mathbb{R}^{n-1}$. Notice that this quantity is independent of $\Omega$ and, hence, well-defined for any point on the surface that is the boundary of the set $E$. Assuming that $\partial E$ is smooth, this curvature converges to the classical mean-curvature [1] in the following sense:

$$
\lim _{\sigma \uparrow 1}(1-\sigma) H_{\sigma}(z)=H(z)
$$


The asymptotics of the fractional perimeter and nonlocal curvature as $\sigma$ goes to zero have also been studied. Namely, it was shown in [10] that

$$
\lim _{\sigma \downarrow 0} \sigma \operatorname{Per}_{\sigma}(E, \Omega)=\frac{1}{\alpha_{n-1}}\left[(1-a(E)) \mathcal{H}^{n}(E \cap \Omega)+a(E) \mathcal{H}^{n}(\Omega \backslash E)\right],
$$

where $a(E):=\lim _{\sigma \downarrow 0} \frac{\sigma}{\omega_{n-1}} \int_{E \backslash B_{1}}|y|^{-n-\sigma} d y$, and in [12] that

$$
\lim _{\sigma \downarrow 0} \sigma H_{\sigma}(z)=\frac{\omega_{n-1}}{\omega_{n-2}} .
$$

The minimizers of the $\sigma$-perimeter functional, called $\sigma$-minimal surfaces, have been studied in great detail in recent years. The regularity of $\sigma$-minimal surfaces has been investigated by Valdinoci and collaborators [6, 13, 15, 19]. Among other things, it is known that $\sigma$-minimal surfaces are smooth off of a singular set of dimension at most $n-8$ for $\sigma$ sufficiently close to 1 . While this is in agreement with a well-known result for classical minimal surfaces [16], $\sigma$-minimal surfaces may have features different from their classical counterparts, in that they may stick to the boundary instead of being orthogonal to it [11, 13. The motion of surfaces by nonlocal mean-curvature has been investigated using level set methods [7, 8, 9, 17].

\subsection{Extension and motivation}

The above discussion of nonlocal mean-curvature applies to surfaces that are the boundary of a set. However, Paroni, Podio-Guidugli, and Seguin discovered that it is possible to define these concepts for any smooth (hyper)surface [18]. The main idea is to define a fractional notion of area and find a condition similar to (5) that a minimizer of this functional must satisfy. Towards this end, they first showed that for a bounded set $E$ with smooth boundary and bounded, open $\Omega$ containing $E$ one can write

$$
\operatorname{Per}_{\sigma}(E, \Omega)=\frac{1}{\alpha_{n-1}} \int_{E} \int_{E^{c}}|x-y|^{-n-\sigma} d x d y=\frac{1}{2 \alpha_{n-1}} \int_{\mathcal{X}(\partial E)}|x-y|^{-n-\sigma} d x d y,
$$

where $\mathcal{X}(\partial E)$ is the set of all pairs $(x, y) \in \mathbb{R}^{n} \times \mathbb{R}^{n}$ such that the oriented line segment connecting $x$ to $y$ crosses $\partial E$ an odd number of times. The validity of (10) follows from the fact that $\mathcal{X}(\partial E)$ and $\left(E \times E^{c}\right) \cup\left(E^{c} \times E\right)$ agree up to a set of $\mathcal{H}^{2 n}$-measure zero. As the far right-hand side of (10) only depends on the surface $\partial E$, and not the set $E$, this motivates the following definition of the $\sigma$-area for a smooth surface $\mathcal{S}$ with or without boundary:

$$
\operatorname{Area}_{\sigma}(\mathcal{S}, \Omega):=\frac{1}{2 \alpha_{n-1}} \int_{\mathcal{X}(\mathcal{S})}|x-y|^{-n-\sigma} \max \left\{\chi_{\Omega}(x), \chi_{\Omega}(y)\right\} d x d y,
$$

where it is assumed that $\mathcal{S}$ is contained in $\Omega$. The presence of $\max \left\{\chi_{\Omega}(x), \chi_{\Omega}(y)\right\}$ in the integrand is necessary to ensure the integral converges. In this way, it is similar to the role 
$\Omega$ plays in the definition of the $\sigma$-perimeter. It follows from (10) that in the case where $\mathcal{S}=\partial E$ and $E \subseteq \Omega$ that $\operatorname{Area}_{\sigma}(\mathcal{S}, \Omega)=\operatorname{Per}_{\sigma}(E, \Omega)$. The $\sigma$-area satisfies a limit relationship analogous to (3). It was shown 18 that if $\mathcal{S}$ minimizes the $\sigma$-area relative to all smooth, bounded, oriented surfaces in $\Omega$ that have the same boundary as $\mathcal{S}$, then $\mathcal{S}$ must satisfy

$$
\int_{\mathcal{A}_{e}(z)}|z-y|^{-n-\sigma} d y-\int_{\mathcal{A}_{i}(z)}|z-y|^{-n-\sigma} d y=0 \quad \text { for all } z \in \mathcal{S},
$$

where

$$
\begin{array}{r}
\mathcal{A}_{e}(z):=\left\{y \in \mathbb{R}^{n} \mid((z, y) \in \mathcal{X}(\mathcal{S}) \text { and }(z-y) \cdot \mathbf{n}(z)>0)\right. \\
\text { or } \left.\left((z, y) \in \mathcal{X}(\mathcal{S})^{c} \text { and }(z-y) \cdot \mathbf{n}(z)<0\right)\right\} \\
\mathcal{A}_{i}(z):=\left\{y \in \mathbb{R}^{n} \mid\left((z, y) \in \mathcal{X}(\mathcal{S})^{c} \text { and }(z-y) \cdot \mathbf{n}(z)>0\right)\right. \\
\text { or }((z, y) \in \mathcal{X}(\mathcal{S}) \text { and }(z-y) \cdot \mathbf{n}(z)<0)\} .
\end{array}
$$

See Figure 1 for a depiction of these sets.

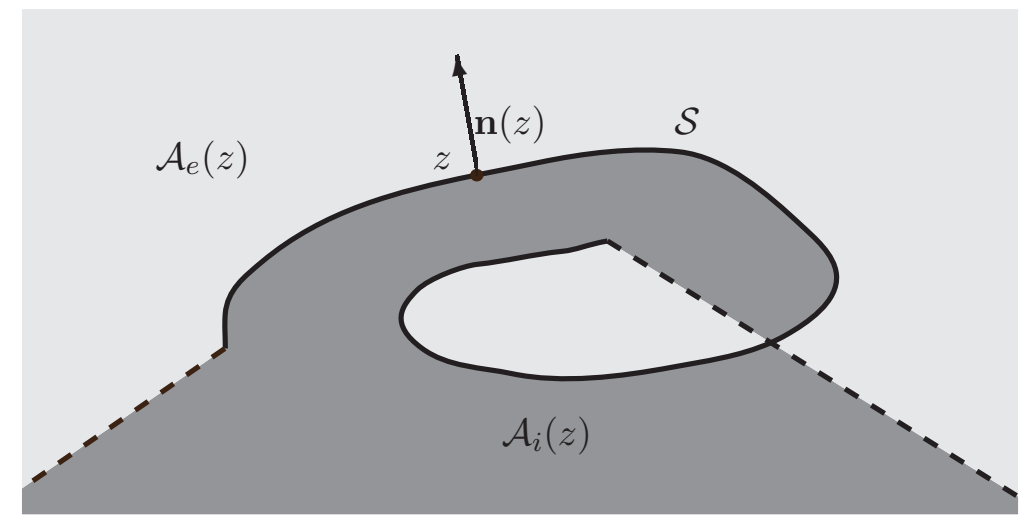

Figure 1: The solid line depicts $\mathcal{S}$. The set of points of density 1 for $\mathcal{A}_{e}(z)$ is shown in light grey, and the set of points of density 1 for $\mathcal{A}_{i}(z)$ is in dark grey. The dashed lines depict the part of the essential boundary between these sets that is not part of $\mathcal{S}$.

This motivates defining the nonlocal mean-curvature of $\mathcal{S}$ at $z$ using the opposite of the left-hand side of (12) - that is,

$$
H_{\sigma}(z):=\frac{1}{\omega_{n-2}} \int_{\mathbb{R}^{n}} \frac{\hat{\chi}_{\mathcal{S}}(z, y)}{|z-y|^{n+\sigma}} d y \quad \text { for all } z \in \mathcal{S},
$$

where

$$
\hat{\chi}_{\mathcal{S}}(z, y):= \begin{cases}1 & y \in \mathcal{A}_{i}(z), \\ 0 & y \notin \mathcal{A}_{i}(z) \cup \mathcal{A}_{e}(z), \\ -1 & y \in \mathcal{A}_{e}(z)\end{cases}
$$


and it is understood the integral is computed as a principle value. The opposite of the left-hand side of (12) is used so that the nonlocal mean-curvature of a sphere with outward orientation is negative, just as in the case for the classical mean-curvature. Notice that $H_{\sigma}$ does not depend on $\Omega$. Unsurprisingly, this curvature satisfies the limit relation (7).

To motivate a definition of fractional length, we will consider the $\sigma$-area in two dimensions, where a hypersurface is a curve. When $n=2$, the $\sigma$-area becomes

$$
\operatorname{Area}_{\sigma}(\mathcal{S}, \Omega)=\frac{1}{4} \int_{\mathcal{X}(\mathcal{S})} \frac{\max \left\{\chi_{\Omega}(x), \chi_{\Omega}(y)\right\}}{|x-y|^{2+\sigma}} d x d y
$$

The domain of integration here consists of line segments that are described by their endpoints. A given line segment connecting $x$ to $y$ can be viewed as a one-dimensional disc and, hence, can be described by its midpoint $p$, a unit vector $\mathbf{u}$ normal to the disc, and a radius $r$ so that

$$
(x, y)=\left(p-r \mathbf{u}^{\prime}, p+r \mathbf{u}^{\prime}\right),
$$

where $\mathbf{u}^{\prime}$ is obtained by rotating $\mathbf{u}$ clockwise by $90^{\circ}$. Utilizing this change of variables, (17) can be rewritten as

$$
\operatorname{Area}_{\sigma}(\mathcal{S}, \Omega)=\frac{1}{2} \int_{\mathcal{D}(\mathcal{S})}(2 r)^{-1-\sigma} \max \left\{\chi_{\Omega}\left(p-r \mathbf{u}^{\prime}\right), \chi_{\Omega}\left(p+r \mathbf{u}^{\prime}\right)\right\} d \mathcal{H}^{4}(p, \mathbf{u}, r),
$$

where $\mathcal{D}(\mathcal{S})$ consists of all triples $(p, \mathbf{u}, r)$ describing those one-dimensional discs that intersect $\mathcal{S}$ an odd number of times and $\mathcal{H}^{4}$ is the 4-dimensional Hausdorff measure. It is this formula for the fractional length that can be generalized to a curve in $n$ dimensions.

Before this generalization is done, we first study the measure theoretic properties of the set of all discs that intersect a curve an odd number of times and other related sets of discs in Section 2. In Section 3 the fractional length is defined and it is shown that it converges, in an appropriate limit, to the classical notion of length up to a multiplicative constant. Next, Section 4 is dedicated to computing the Euler-Lagrange equation associated with the fractional length and this result is used to motivate a definition of nonlocal curvature for a curve. The Appendix contains several change of variables formulas that are useful in established the desired results.

\section{Sets of discs}

In this section the set of all $(n-1)$-dimensional discs, and various subsets of it, are studied in $\mathbb{R}^{n}$, with $n \geq 2$. The results established here make precise which discs are integrated over in the definition of the nonlocal length. Moreover, they will be crucial in computing

the first variation of the fractional length. We use $\mathcal{U}_{n}$ to denote the set of unit vectors in $\mathbb{R}^{n}$, and set

$$
\mathcal{U}_{\perp}^{2}:=\left\{(\mathbf{a}, \mathbf{b}) \in \mathcal{U}_{n} \times \mathcal{U}_{n} \mid \mathbf{a} \cdot \mathbf{b}=0\right\},
$$


which consists of all pairs of orthogonal unit vectors.

The $(n-1)$-dimensional disc with center $p$, normal unit-vector $\mathbf{u}$, and radius $r$ is denoted by

$$
D(p, \mathbf{u}, r):=\left\{p+\xi \mathbf{v} \mid(\mathbf{u}, \mathbf{v}) \in \mathcal{U}_{\perp}^{2}, \xi \in[0, r)\right\} .
$$

By the boundary $\partial D(p, \mathbf{u}, r)$ of one of these discs we mean the $(n-2)$-dimensional manifold

$$
\left\{p+r \mathbf{v} \in \mathbb{R}^{n} \mid \mathbf{v} \in \mathcal{U}_{n} \cap\{\mathbf{u}\}^{\perp}\right\},
$$

where $\{\mathbf{u}\}^{\perp}$ is the set of all vectors orthogonal to $\mathbf{u}$. The disc together with its boundary is denoted by $\bar{D}(p, \mathbf{u}, r)$. Thus, the set of all discs in $\mathbb{R}^{n}$ can be described by elements $(p, \mathbf{u}, r)$ of the set $\mathcal{D}:=\mathbb{R}^{n} \times \mathcal{U}_{n} \times \mathbb{R}^{+}$. For this reason, we will sometimes refer to the elements of $\mathcal{D}$ as discs.

Consider a $C^{1}$ curve $\mathcal{C}$ in $\mathbb{R}^{n}$. We will assume that its closure $\overline{\mathcal{C}}$ is a one-dimensional manifold with boundary $\partial \mathcal{C}$, which consists of either two points or is empty. Orient $\mathcal{C}$ so that at each point $z \in \mathcal{C}$ we have a unit tangent $\mathbf{t}(z)$. Consider the following subsets of the set of all discs $\mathcal{D}$ :

$$
\begin{aligned}
\mathcal{D}_{\partial \mathcal{C}} & :=\left\{(p, \mathbf{u}, r) \in \mathcal{D} \mid \mathcal{H}^{0}(\bar{D}(p, \mathbf{u}, r) \cap \partial \mathcal{C}) \neq 0\right\} \\
\mathcal{D}_{\partial \mathcal{C} 2} & :=\left\{(p, \mathbf{u}, r) \in \mathcal{D} \mid \mathcal{H}^{0}(\bar{D}(p, \mathbf{u}, r) \cap \partial \mathcal{C})=2\right\} \\
\mathcal{D}_{\text {tan }} & :=\{(p, \mathbf{u}, r) \in \mathcal{D} \mid \text { there is a } z \in \bar{D}(p, \mathbf{u}, r) \cap \mathcal{C} \text { such that } \mathbf{t}(z) \cdot \mathbf{u}=0\}, \\
\mathcal{D}_{\infty} & :=\left\{(p, \mathbf{u}, r) \in \mathcal{D} \mid \mathcal{H}^{0}(\bar{D}(p, \mathbf{u}, r) \cap \mathcal{C})=\infty\right\} \\
\mathcal{D}_{\partial 1} & :=\left\{(p, \mathbf{u}, r) \in \mathcal{D} \mid \mathcal{H}^{0}(\partial D(p, \mathbf{u}, r) \cap \mathcal{C})=1\right\} \\
\mathcal{D}_{\partial 2} & :=\left\{(p, \mathbf{u}, r) \in \mathcal{D} \mid \mathcal{H}^{0}(\partial D(p, \mathbf{u}, r) \cap \mathcal{C}) \geq 2\right\} \\
\mathcal{D}_{\partial} & :=\mathcal{D}_{\partial 1} \cup \mathcal{D}_{\partial 2}, \\
\mathcal{D}_{\text {odd }} & :=\left\{(p, \mathbf{u}, r) \in \mathcal{D} \backslash\left(\mathcal{D}_{\partial \mathcal{C}} \cup \mathcal{D}_{\tan } \cup \mathcal{D}_{\partial}\right) \mid \mathcal{H}^{0}(D(p, \mathbf{u}, r) \cap \mathcal{C}) \text { is an odd number }\right\}, \\
\mathcal{D}_{\text {even }} & :=\left\{(p, \mathbf{u}, r) \in \mathcal{D} \backslash\left(\mathcal{D}_{\partial \mathcal{C}} \cup \mathcal{D}_{\tan } \cup \mathcal{D}_{\partial}\right) \mid \mathcal{H}^{0}(D(p, \mathbf{u}, r) \cap \mathcal{C}) \text { is an even number }\right\} .
\end{aligned}
$$

The following lemma discusses the measure theoretic properties of these sets.

Lemma 2.1. The following facts are true:

1. $\mathcal{H}^{2 n-1}\left(\mathcal{D}_{\partial \mathcal{C}} \cap \mathcal{E}\right)<\infty$ for any bounded, open set $\mathcal{E} \subseteq \mathcal{D}$,

2. $\mathcal{H}^{2 n-1}\left(\mathcal{D}_{\tan } \cap \mathcal{E}\right)<\infty$ for any bounded, open set $\mathcal{E} \subseteq \mathcal{D}$,

3. $\mathcal{H}^{2 n-1}\left(\mathcal{D}_{\partial} \cap \mathcal{E}\right)<\infty$ for any bounded, open set $\mathcal{E} \subseteq \mathcal{D}$,

4. $\mathcal{D}_{\infty} \subseteq \mathcal{D}_{\tan }$,

5. $\mathcal{H}^{2 n-1}\left(\mathcal{D}_{\partial 2}\right)=\mathcal{H}^{2 n-1}\left(\mathcal{D}_{\partial \mathcal{C} 2}\right)=0$,

6. $\mathcal{H}^{2 n-1}\left(\mathcal{D}_{\partial \mathcal{C}} \cap \mathcal{D}_{\partial}\right)=0$, 
7. $\mathcal{D}_{\text {even }}$ and $\mathcal{D}_{\text {odd }}$ are open subsets of $\mathcal{D}$,

8. $\mathcal{D}=\mathcal{D}_{\text {odd }} \cup \mathcal{D}_{\text {even }} \cup \mathcal{D}_{\partial \mathcal{C}} \cup \mathcal{D}_{\text {tan }} \cup \mathcal{D}_{\partial}$.

Proof. Let $\mathcal{E}$ be a bounded, open subset of $\mathcal{D}$. Find $R>0$ such that if $(p, \mathbf{u}, r) \in \mathcal{E}$, then $r \in(0, R]$. Set $\mathcal{E}_{\Xi}=\Xi^{-1}(\mathcal{E})$ and $\mathcal{E}_{\Psi}=\Psi^{-1}(\mathcal{E})$, where $\Xi$ and $\Psi$ are defined in (95) and (97), respectively.

Item 10 Consider the set

$$
\mathcal{A}_{\partial \mathcal{C}}:=\partial \mathcal{C} \times \mathcal{U}_{\perp}^{2} \times \mathbb{R}^{+} \times \mathbb{R}^{+}
$$

and the function $\Xi: \mathcal{A}_{\partial \mathcal{C}} \rightarrow \mathcal{D}$ defined by (95) in the Appendix. Notice that $\mathcal{D}_{\partial \mathcal{C}} \cap \mathcal{E} \subseteq$ $\Xi\left(\mathcal{A}_{\partial \mathcal{C}} \cap \mathcal{E}_{\Xi}\right)$. Since $\Xi$ is Lipschitz on $\mathcal{A}_{\partial \mathcal{C}} \cap \mathcal{E}_{\Xi}$ and $\mathcal{H}^{2 n-1}\left(\mathcal{A}_{\partial \mathcal{C}} \cap \mathcal{E}_{\Xi}\right)<\infty$, it follows that $\mathcal{H}^{2 n-1}\left(\mathcal{D}_{\partial \mathcal{C}} \cap \mathcal{E}\right)<\infty$.

Item 21) The proof is the same as Item 1 with the exception that one uses the set

$$
\mathcal{A}_{\tan }:=\bigcup_{z \in \mathcal{C}}\{z\} \times\left\{(\mathbf{a}, \mathbf{b}) \in \mathcal{U}_{\perp}^{2} \mid \mathbf{b} \cdot \mathbf{t}(z)=0\right\} \times \mathbb{R}^{+} \times \mathbb{R}^{+}
$$

rather than $\mathcal{A}_{\partial \mathcal{C}}$.

Item 3) Consider the set

$$
\mathcal{A}_{\partial}:=\mathcal{C} \times \mathcal{U}_{\perp}^{2} \times \mathbb{R}^{+}
$$

and the function $\Psi: \mathcal{A}_{\partial} \rightarrow \mathcal{D}$ defined by (97) in the Appendix. Notice that $\mathcal{D}_{\partial} \cap \mathcal{E} \subseteq$ $\Psi\left(\mathcal{A}_{\partial} \cap \mathcal{E}_{\Psi}\right)$. Since $\Psi$ is Lipschitz on $\mathcal{A}_{\partial} \cap \mathcal{E}_{\Psi}$ and $\mathcal{H}^{2 n-1}\left(\mathcal{A}_{\partial} \cap \mathcal{E}_{\Psi}\right)<\infty$, it follows that $\mathcal{H}^{2 n-1}\left(\mathcal{D}_{\partial} \cap \mathcal{E}\right)<\infty$.

Item (4) Consider $(p, \mathbf{u}, r) \in \mathcal{D}_{\infty}$, so that there are an infinite number of points in $\bar{D}(p, \mathbf{u}, r) \cap \mathcal{C}$. Since this set is compact it follows that this intersection has a cluster point, say $z \in \bar{D}(p, \mathbf{u}, r) \cap \mathcal{C}$. Suppose that $(p, \mathbf{u}, r) \notin \mathcal{D}_{\text {tan }}$, so that $\mathbf{t}(z) \cdot \mathbf{u} \neq 0$. Since in a neighborhood of $z$ the curve $\mathcal{C}$ can be approximated by its tangent line which has direction $\mathbf{t}(z)$, it follows that there are no points in this neighborhood besides $z$ in the intersection $\bar{D}(p, \mathbf{u}, r) \cap \mathcal{C}$. This contradicts the fact that $z$ is a cluster point of $\bar{D}(p, \mathbf{u}, r) \cap \mathcal{C}$. Thus, we must have $\mathbf{t}(z) \cdot \mathbf{u}=0$, so $z \in \mathcal{D}_{\text {tan }}$.

Item 5) Consider the set

$$
\mathcal{A}_{\partial 2}:=\left\{\left(z_{1}, z_{2}, \mathbf{a}, \mathbf{b}\right) \in \mathcal{C} \times \mathcal{C} \times \mathcal{U}_{\perp}^{2} \mid \mathbf{a} \cdot\left(z_{2}-z_{1}\right)>0, \mathbf{b} \cdot\left(z_{2}-z_{1}\right)=0\right\} .
$$

and define the function $\Lambda: \mathcal{A}_{\partial 2} \rightarrow \mathcal{D}$ by

$$
\Lambda\left(z_{1}, z_{2}, \mathbf{a}, \mathbf{b}\right):=\left(z_{1}+\frac{\left|z_{2}-z_{1}\right|^{2}}{2 \mathbf{a} \cdot\left(z_{2}-z_{1}\right)} \mathbf{a}, \mathbf{b}, \frac{\left|z_{2}-z_{1}\right|^{2}}{2 \mathbf{a} \cdot\left(z_{2}-z_{1}\right)}\right) .
$$

One can check that the boundary of the disc $D\left(\Lambda\left(z_{1}, z_{2}, \mathbf{a}, \mathbf{b}\right)\right)$ intersects $\mathcal{C}$ at $z_{1}$ and $z_{2}$. Thus, $\mathcal{D}_{\partial 2} \subseteq \Lambda\left(\mathcal{A}_{\partial 2}\right)$. Moreover, $\Lambda$ is locally Lipschitz on $\mathcal{A}_{\partial 2}$. It follows that since $\mathcal{H}^{2 n-1}\left(\mathcal{A}_{\partial 2}\right)=0$, we must have $\mathcal{H}^{2 n-1}\left(\mathcal{D}_{\partial 2}\right)=0$. 
To establish the second part of this item, first note that it is trivially true if $\partial \mathcal{C}$ is empty. Now assume that $q_{1}$ and $q_{2}$ are the two endpoints of $\mathcal{C}$. Define

$$
\mathcal{A}_{\partial \mathcal{C} 2}:=\partial \mathcal{C} \times\left\{(\mathbf{a}, \mathbf{b}) \in \mathcal{U}_{\perp}^{2} \mid \mathbf{b} \cdot\left(q_{1}-q_{2}\right)=0\right\} \times \mathbb{R}^{+} \times \mathbb{R}^{+} .
$$

Notice that $\mathcal{D}_{\partial \mathcal{C} 2} \subseteq \Xi\left(\mathcal{A}_{\partial \mathcal{C} 2}\right)$. Since $\Xi$ is locally Lipschitz on $\mathcal{A}_{\partial \mathcal{C}}$ and $\mathcal{H}^{2 n-1}\left(\mathcal{A}_{\partial \mathcal{C} 2}\right)=0$, it follows that $\mathcal{H}^{2 n-1}\left(\mathcal{D}_{\partial \mathcal{C} 2}\right)=0$.

Item [6) Consider the set

$$
\mathcal{A}_{\partial \mathcal{C} \partial}:=\left\{(q, z, \mathbf{a}, \mathbf{b}, r) \in \partial \mathcal{C} \times \mathcal{A}_{\partial} \mid(q-z) \cdot \mathbf{b}=0\right\}
$$

and the function $\Psi_{\partial}: \mathcal{A}_{\partial \mathcal{C} \partial} \rightarrow \mathcal{D}$ defined by

$$
\Psi_{\partial}(q, z, \mathbf{a}, \mathbf{b}, r):=\Psi(z, \mathbf{a}, \mathbf{b}, r) \quad \text { for all }(q, z, \mathbf{a}, \mathbf{b}, r) \in \mathcal{A}_{\partial \mathcal{C} \partial} .
$$

Notice that $\mathcal{D}_{\partial \mathcal{C}} \cap \mathcal{D}_{\partial} \subseteq \Psi_{\partial}\left(\mathcal{A}_{\partial \mathcal{C} \partial}\right)$ and $\mathcal{H}^{2 n-1}\left(\mathcal{A}_{\partial \mathcal{C} \partial}\right)=0$. It follows that $\mathcal{H}^{2 n-1}\left(\mathcal{D}_{\partial \mathcal{C}} \cap \mathcal{D}_{\partial}\right)=$ 0 .

Item [7) This is clear from the definitions of $\mathcal{D}_{\text {even }}$ and $\mathcal{D}_{\text {odd }}$.

Item (8) This follows from the definitions of the various sets involved.

The previous result yielded enough information to obtain the properties of $\mathcal{D}_{\text {odd }}$ we require.

Proposition 2.2. The set $\mathcal{D}_{\text {odd }}$ is locally of finite perimeter. Moreover, the essential boundary $\partial^{*} \mathcal{D}_{\text {odd }}$ of this set is equal to $\mathcal{D}_{\partial \mathcal{C}} \cup \mathcal{D}_{\partial 1}$ up to a set of $\mathcal{H}^{2 n-1}$-measure zero.

Proof. From Items 1 and 8 of Lemma 2.1, we see that $\partial^{*} \mathcal{D}_{\text {odd }} \subseteq \mathcal{D}_{\partial \mathcal{C}} \cup \mathcal{D}_{\text {tan }} \cup \mathcal{D}_{\partial}$. Thus, from Items 13 of the same lemma, whenever $\mathcal{E} \subseteq \mathcal{D}$ is a bounded, open set we have

$$
\mathcal{H}^{2 n-1}\left(\partial^{*} \mathcal{D}_{\text {odd }} \cap \mathcal{E}\right) \leq \mathcal{H}^{2 n-1}\left(\left(\mathcal{D}_{\partial \mathcal{C}} \cup \mathcal{D}_{\tan } \cup \mathcal{D}_{\partial}\right) \cap \mathcal{E}\right)<\infty .
$$

By a result of Federer, see 4.5.11 of [14], we can conclude that $\mathcal{D}_{\text {odd }}$ has finite perimeter in $\mathcal{E}$. Moreover, it is known, see Ambrosio, Fusco, and Pallara [2] Theorem 3.61, that it follows that $\mathcal{D}_{\text {odd }}$ has density either $0,1 / 2$, or 1 at $\mathcal{H}^{2 n-1}$-a.e. point of $\mathcal{E}$, and $\partial^{*} \mathcal{D}_{\text {odd }} \cap \mathcal{E}$ consists of those points with density $1 / 2$ up to a set of $\mathcal{H}^{2 n-1}$-measure zero.

We first show that $\mathcal{D}_{\text {tan }} \backslash\left(\mathcal{D}_{\partial \mathcal{C}} \cup \mathcal{D}_{\partial}\right)$ has density either 0 or 1 relative to $\mathcal{D}_{\text {odd }}$ at $\mathcal{H}^{2 n-1}$-a.e. point and, hence, cannot be part of $\partial^{*} \mathcal{D}_{\text {odd }}$. Fix $(p, \mathbf{u}, r) \in \mathcal{D}_{\tan } \backslash\left(\mathcal{D}_{\partial \mathcal{C}} \cup \mathcal{D}_{\partial}\right)$. Find a small, connected neighborhood $\mathcal{N}$ of $(p, \mathbf{u}, r)$ in $\mathcal{D}$ that is disjoint from $\mathcal{D}_{\partial}$ and $\mathcal{D}_{\partial \mathcal{C}}$, which is possible since these two sets are closed in $\mathcal{D}$. From Item 2 of Lemma 2.1, for $\mathcal{H}^{2 n}$ a.e. $\left(p^{\prime}, \mathbf{u}^{\prime}, r^{\prime}\right) \in \mathcal{N}$, the disc $D\left(p^{\prime}, \mathbf{u}^{\prime}, r^{\prime}\right)$ is not tangent to $\mathcal{C}$ and from Item 4 such discs only intersect $\mathcal{C}$ a finite number of times. Consider two such discs $\left(p_{1}, \mathbf{u}_{1}, r_{1}\right),\left(p_{2}, \mathbf{u}_{2}, r_{2}\right) \in \mathcal{N}$. Since $\mathcal{N}$ is a small neighborhood, we know that the $\operatorname{discs} D\left(p_{1}, \mathbf{u}_{1}, r_{1}\right)$ and $D\left(p_{2}, \mathbf{u}_{2}, r_{2}\right)$

\footnotetext{
${ }^{1}$ For the definition of sets of finite perimeter and essential boundary see, for example, Ambrosio, Fusco, and Pallara [2].
} 
are close in the sense that their centers are close, their orientations are close, and their radii are close. Since $\mathcal{N}$ is connected, there is a continuous path in $\mathcal{N}$ from $\left(p_{1}, \mathbf{u}_{1}, r_{1}\right)$ to $\left(p_{2}, \mathbf{u}_{2}, r_{2}\right)$. In the process of going along this path, the disc $D\left(p_{1}, \mathbf{u}_{1}, r_{1}\right)$ sweeps out a tube in $\mathbb{R}^{n}$ until it reaches $D\left(p_{2}, \mathbf{u}_{2}, r_{2}\right)$. Let $\mathcal{T}$ denote this tube. The boundary of $\mathcal{T}$ consists of the two discs $D\left(p_{1}, \mathbf{u}_{1}, r_{1}\right)$ and $D\left(p_{2}, \mathbf{u}_{2}, r_{2}\right)$ along with the side of the tube, which is obtained by starting with $\partial D\left(p_{1}, \mathbf{u}_{1}, r_{1}\right)$ and proceeding along the path of discs until one reaches $\partial D\left(p_{2}, \mathbf{u}_{2}, r_{2}\right)$. Let $\mathcal{S}$ denote the side of the tube. Since $\mathcal{N}$ is disjoint from $\mathcal{D}_{\partial}$ and $\mathcal{D}_{\partial \mathcal{C}}$, it follows that

$$
\mathcal{T} \cap \partial \mathcal{C}=\emptyset \quad \text { and } \quad \mathcal{S} \cap \mathcal{C}=\emptyset .
$$

We proceed by showing that $\mathcal{H}^{0}\left(D\left(p_{1}, \mathbf{u}_{1}, r_{1}\right) \cap \mathcal{C}\right)$ and $\mathcal{H}^{0}\left(D\left(p_{2}, \mathbf{u}_{2}, r_{2}\right) \cap \mathcal{C}\right)$ have the same parity, meaning that they are either both even or both odd, by showing that their sum is even. If both of these numbers are zero, we are done. Now suppose that after starting at one of the endpoints of $\mathcal{C}$ and going along the curve, it intersects one of the discs. As the two discs are part of the boundary of the tube $\mathcal{T}$, this means that after this intersection the curve has either entered or left the tube. The latter option is not possible since this would mean that the curve would have entered the tube previously through the side $\mathcal{S}$, which would violate $(30)_{2}$. After the curve intersects one of the discs and is inside $\mathcal{T}$, it cannot end inside $\mathcal{T}$ as this would violate $(30) 1$. Thus, the curve must leave $\mathcal{T}$. This can be accomplished by either crossing one of the two discs or by leaving $\mathcal{T}$ through the side of the tube $\mathcal{S}$. However, this last option is not possible as it violates $(30)_{2}$. Thus, the curve must intersect one of the discs to exit $\mathcal{T}$. After the curve leaves the tube $\mathcal{T}$, this argument can be repeated again and again until the other endpoint is reached. Regardless of how many times this is repeated, the total number of intersections the curve has with $D\left(p_{1}, \mathbf{u}_{1}, r_{1}\right)$ and $D\left(p_{2}, \mathbf{u}_{2}, r_{2}\right)$ is even. Thus, $\mathcal{H}^{0}\left(D\left(p_{1}, \mathbf{u}_{1}, r_{1}\right) \cap \mathcal{C}\right)$ and $\mathcal{H}^{0}\left(D\left(p_{2}, \mathbf{u}_{2}, r_{2}\right) \cap \mathcal{C}\right)$ have the same parity. If the parity is even, then almost all discs in $\mathcal{N}$ are also in $\mathcal{D}_{\text {even }}$, and so the density of $\mathcal{D}_{\text {odd }}$ at $(p, \mathbf{u}, r)$ is 0 , while if the parity is odd, then it is 1 .

We conclude that $\partial^{*} \mathcal{D}_{\text {odd }} \cap \mathcal{E} \subseteq\left(\mathcal{D}_{\partial \mathcal{C}} \cup \mathcal{D}_{\partial}\right) \cap \mathcal{E}$ up to a set of $\mathcal{H}^{2 n-1}$-measure zero. Item 5 of the lemma says that $\mathcal{H}^{2 n-1}\left(\mathcal{D}_{\partial 2}\right)=0$ and, so, $\partial^{*} \mathcal{D}_{\text {odd }} \cap \mathcal{E} \subseteq\left(\mathcal{D}_{\partial \mathcal{C}} \cup \mathcal{D}_{\partial 1}\right) \cap \mathcal{E}$ up to a set of $\mathcal{H}^{2 n-1}$-measure zero.

Next we establish that $\left(\mathcal{D}_{\partial 1} \backslash \mathcal{D}_{\partial \mathcal{C}}\right) \cap \mathcal{E} \subseteq \partial^{*} \mathcal{D}_{\text {odd }} \cap \mathcal{E}$ by arguing that all points $(p, \mathbf{u}, r) \in$ $\left(\mathcal{D}_{\partial 1} \backslash \mathcal{D}_{\partial \mathcal{C}}\right) \cap \mathcal{E}$ have density $1 / 2$ relative to $\mathcal{D}_{\text {odd }}$. First notice that $\mathcal{D}_{\partial}=\Psi\left(\mathcal{A}_{\partial}\right)$, where $\mathcal{A}_{\partial}$ is defined in (23) and $\Psi$ is the function defined in (97) of the Appendix. This means that $\mathcal{D}_{\partial}$ is an immersed submanifold of $\mathcal{D}$. Moreover, the function $\Psi$ is an embedding on the preimage of $\mathcal{D}_{\partial 1}$ under $\Psi$ and, so, $\mathcal{D}_{\partial 1}$ is a $(2 n-1)$-dimensional embedded submanifold of $\mathcal{D}$. It follows from the definitions of the sets involved that if $(p, \mathbf{u}, r) \in \mathcal{D}_{\partial 1} \backslash \mathcal{D}_{\partial \mathcal{C}}$, then any neighborhood of $(p, \mathbf{u}, r)$ in $\mathcal{D}$ contains elements of $\mathcal{D}_{\text {odd }}$ and $\mathcal{D}_{\text {even. }}$ Putting this together with the fact that $\mathcal{D}_{\partial 1}$ is a $(2 n-1)$-dimensional embedded submanifold of $\mathcal{D}$ and $\mathcal{D}_{\partial \mathcal{C}}$ is a closed set, we can conclude that the density at $(p, \mathbf{u}, r)$ of $\mathcal{D}_{\text {odd }}$ must be $1 / 2$.

The proof that $\left(\mathcal{D}_{\partial \mathcal{C}} \backslash \mathcal{D}_{\partial 1}\right) \cap \mathcal{E} \subseteq \partial^{*} \mathcal{D}_{\text {odd }} \cap \mathcal{E}$ uses a similar argument and, so, will be skipped. It then follows from Items 5 and 6 of Lemma 2.1 that $\left(\mathcal{D}_{\partial \mathcal{C}} \cup \mathcal{D}_{\partial 1}\right) \cap \mathcal{E} \subseteq$ 
$\partial^{*} \mathcal{D}_{\text {odd }} \cap \mathcal{E}$.

Since $\mathcal{D}_{\text {odd }}$ is locally a set of finite perimeter, it has an exterior unit normal at $\mathcal{H}^{2 n-1}$ a.e. point of its essential boundary. The next result describes this normal vector along the part of $\partial^{*} \mathcal{D}_{\text {odd }}$ that we will need later.

Proposition 2.3. For $\mathcal{H}^{2 n-1}$-a.e. $(p, \mathbf{u}, r) \in \partial^{*} \mathcal{D}_{\text {odd }}$ such that $D(p, \mathbf{u}, r) \cap \partial \mathcal{C}$ is empty, there is a unique $z \in \partial D(p, \mathbf{u}, r) \cap \mathcal{C}$. Moreover, for such $(p, \mathbf{u}, r)$ the exterior unit-normal $\boldsymbol{\nu}(p, \mathbf{u}, r) \in \mathbb{R}^{n} \times\{\mathbf{u}\}^{\perp} \times \mathbb{R}$ is given by

$$
\boldsymbol{\nu}(p, \mathbf{u}, r):= \begin{cases}\mathbf{m}(p, \mathbf{u}, r) & \text { if } \mathcal{H}^{0}(D(p, \mathbf{u}, r) \cap \mathcal{C} \text { is an odd number } \\ -\mathbf{m}(p, \mathbf{u}, r) & \text { if } \mathcal{H}^{0}(D(p, \mathbf{u}, r) \cap \mathcal{C} \text { is an even number }\end{cases}
$$

where

$$
\mathbf{m}(p, \mathbf{u}, r):=\frac{\left(z-p+\frac{(p-z) \cdot \mathbf{t}}{\mathbf{u} \cdot \mathbf{t}} \mathbf{u}, \frac{(p-z) \cdot \mathbf{t}}{\mathbf{u} \cdot \mathbf{t}}(p-z), r\right)}{\sqrt{|p-z|^{2}+\left(\frac{(p-z) \cdot \mathbf{t}}{\mathbf{u} \cdot \mathbf{t}}\right)^{2}|z-p|^{2}+\left(\frac{(p-z) \cdot \mathbf{t}}{\mathbf{u} \cdot \mathbf{t}}\right)^{2}+r^{2}}}
$$

and $\mathbf{t}$ is the tangent to $\mathcal{C}$ at $z$.

Proof. We are looking for the exterior unit-normal to $\mathcal{D}_{\text {odd }}$ on $\partial^{*} \mathcal{D}_{\text {odd }} \backslash \mathcal{D}_{\partial \mathcal{C}}$. By Proposition 2.2 and Item 6 of Lemma 2.1] it suffices to find the exterior unit-normal on

$$
\mathcal{P}:=\partial^{*} \mathcal{D}_{\text {odd }} \cap\left(\mathcal{D}_{\partial 1} \backslash \mathcal{D}_{\partial \mathcal{C}}\right) .
$$

As argued before, $\mathcal{D}_{\partial 1}$ is an embedded submanifold of $\mathcal{D}$ and, thus, is $\mathcal{H}^{2 n-1}$-rectifiable. It follows that the approximate tangent space to $\mathcal{P}$, where it exists, coincides with the tangent space of $\mathcal{D}_{\partial 1}$. Thus, to calculate the exterior unit-normal on $\mathcal{P}$, we first find the tangent space to $\mathcal{D}_{\partial 1}$ at any point.

Let $(p, \mathbf{u}, r) \in \mathcal{P}$ and find the unique $(z, \mathbf{a}, \mathbf{b}, r) \in \mathcal{A}_{\partial}$, see (23), that gets mapped to $(p, \mathbf{u}, r)$ under $\Psi$, which is defined in (97). Denote by $\mathbf{t}$ the unit tangent vector to $\mathcal{C}$ at $z$. Since $(p, \mathbf{u}, r) \notin \mathcal{D}_{\text {tan }}$, we must have $\mathbf{b} \cdot \mathbf{t} \neq 0$. A curve in $\mathcal{A}_{\partial}$ that passes through $(z, \mathbf{a}, \mathbf{b}, r)$ induces, via the mapping $\Psi$, a curve in $\mathcal{D}_{\partial 1}$ that passes through $(p, \mathbf{u}, r)$. By differentiating such curves we can generate vectors in the tangent space of $\mathcal{D}_{\partial 1}$ at $(p, \mathbf{u}, r)$. In particular, one can find that the following vectors are in the tangent space:

$$
(\mathbf{t}, \mathbf{0}, 0), \quad(\mathbf{c}, \mathbf{0}, 0), \quad(\mathbf{0}, \mathbf{d}, 0), \quad(\mathbf{a}, 0,1), \quad(r \mathbf{b},-\mathbf{a}, 0)
$$

where $\mathbf{c}$ and $\mathbf{d}$ are any vectors orthogonal to both $\mathbf{a}$ and $\mathbf{b}$. This generates a list of $2 n-1$ linearly independent vectors since $\mathbf{b} \cdot \mathbf{t} \neq 0$. Thus, these vectors span the tangent space at $(p, \mathbf{u}, r)$. A vector in $\mathbb{R}^{n} \times\{\mathbf{u}\}^{\perp} \times \mathbb{R}$, which is the tangent space to $\mathcal{D}$ at $(p, \mathbf{u}, r)$, that is orthogonal to the list of vectors in (34) is

$$
\left(-\mathbf{a}+\frac{\mathbf{a} \cdot \mathbf{t}}{\mathbf{b} \cdot \mathbf{t}} \mathbf{b}, r \frac{\mathbf{a} \cdot \mathbf{t}}{\mathbf{b} \cdot \mathbf{t}} \mathbf{b}, 1\right)
$$


Since $(p, \mathbf{u}, r)=\Psi(z, \mathbf{a}, \mathbf{b}, r)=(z+r \mathbf{a}, \mathbf{b}, r)$, we can replace $\mathbf{a}$ with $(p-z) / r$ and $\mathbf{b}$ with u. Doing so and normalizing this vector results in the vector $\mathbf{m}$ defined in (32).

The vector $\mathbf{m}$ at $(p, \mathbf{u}, r)$ is pointing outward from $\mathcal{D}_{\text {odd }}$ if the interior of the disc associated with $(p, \mathbf{u}, r)$ crosses $\mathcal{C}$ an odd number of times. To see this, let $\gamma$ be a smooth curve in $\mathcal{D}$ defined on an interval of $\mathbb{R}$ containing zero such that $\gamma(0)=(p, \mathbf{u}, r)$ and $\gamma^{\prime}(0)=\mathbf{m}$. For small negative values of $t$, we have $\gamma(t) \in \mathcal{D}_{\text {odd }}$ since the last component of $\mathbf{m}$ is positive and so the disc $D(\gamma(t))$ will intersect the curve an odd number of times and its boundary will not intersect the curve. Moreover, $\gamma(t) \notin \mathcal{D}_{\text {odd }}$ for small positive $t$ because the curve $\mathcal{C}$ will cross the disc $D(\gamma(t))$ one more time than the disc $D(p, \mathbf{u}, r)$ since the boundary of this disc intersects the curve. Using similar logic, one can see that $\mathbf{- m}$ is the outward normal if $D(p, \mathbf{u}, r) \cap \mathcal{C}$ has an even number of points.

In the next section we will use the notation $\mathcal{D}(\mathcal{C}):=\mathcal{D}_{\text {odd }}$ to highlight that this set of discs depends on the curve $\mathcal{C}$. The previous two results describe $\partial^{*} \mathcal{D}(\mathcal{C})$, but we have to go one step further and understand the boundaries of the sets $\mathcal{D}_{\Omega}$ and $\mathcal{D}_{\Omega}(\mathcal{C})$, which are defined by

$$
\mathcal{D}_{\Omega}:=\{(p, \mathbf{u}, r) \in \mathcal{D} \mid \partial D(p, \mathbf{u}, r) \cap \Omega \neq \emptyset\} \quad \text { and } \quad \mathcal{D}_{\Omega}(\mathcal{C}):=\mathcal{D}(\mathcal{C}) \cap \mathcal{D}_{\Omega},
$$

where $\Omega \subseteq \mathbb{R}^{n}$ is an open, bounded set with smooth boundary.

Proposition 2.4. The set $\mathcal{D}_{\Omega}$ is locally of finite perimeter. If $(p, \mathbf{u}, r) \in \partial^{*} \mathcal{D}_{\Omega}$, then there is a point on the boundary of $D(p, \mathbf{u}, r)$ that is tangent to $\partial \Omega$. Let $\mathbf{n}_{\Omega}$ is the exterior unit normal defined on the boundary $\partial \Omega$ of $\Omega$. We can write $\partial^{*} \mathcal{D}_{\Omega}=\Psi\left(\mathcal{A}_{\partial \Omega}\right)$ up to a set of $\mathcal{H}^{2 n-1}$-measure zero, where

$$
\mathcal{A}_{\partial \Omega}:=\bigcup_{z \in \partial \Omega}\{z\} \times\left\{(\mathbf{a}, \mathbf{b}) \in \mathcal{U}_{\perp}^{2} \mid \mathbf{n}(z) \cdot \mathbf{u}=0 \text { for all } \mathbf{u} \in\{\mathbf{a}\}^{\perp} \cap\{\mathbf{b}\}^{\perp}\right\} \times \mathbb{R}^{+}
$$

and $\Psi$ is defined in (97). Moreover, the set of $(p, \mathbf{u}, r)$ for which there are two points on the boundary $\partial D(p, \mathbf{u}, r)$ that are tangent to $\partial \Omega$ has $\mathcal{H}^{2 n-1}$-measure zero.

The proof of this involves the same techniques used to establish the other results in this section and, so, will be skipped. Since $\mathcal{D}_{\partial 1} \subseteq \mathcal{D}_{\Omega}$, as $\Omega$ contains $\mathcal{C}$, it follows from Proposition 2.2 that

$$
\partial^{*} \mathcal{D}_{\Omega}(\mathcal{C})=\left(\mathcal{D}_{\partial \mathcal{C}} \cap \mathcal{D}_{\Omega}\right) \cup \mathcal{D}_{\partial 1} \cup \partial^{*} \mathcal{D}_{\Omega},
$$

where this equality holds up to a set of $\mathcal{H}^{2 n-1}$-measure zero. Notice that if we set

$$
\mathcal{A}_{\Omega \partial \mathcal{C}}=\left\{(q, z, \mathbf{a}, \mathbf{b}, r) \in \partial \mathcal{C} \times \mathcal{A}_{\partial \Omega} \mid(z-q) \cdot \mathbf{b}=0 \text { and }|z+r \mathbf{a}-q|<r\right\},
$$

then $\mathcal{D}_{\partial \mathcal{C}} \cap \mathcal{D}_{\Omega}=\Xi\left(\mathcal{A}_{\Omega \partial \mathcal{C}}\right)$ up to a set of $\mathcal{H}^{2 n-1}$-measure zero. Thus, using (23) and Proposition 2.4, the equality (38) can also be written as

$$
\partial^{*} \mathcal{D}_{\Omega}(\mathcal{C})=\Xi\left(\mathcal{A}_{\Omega \partial \mathcal{C}}\right) \cup \Psi\left(\mathcal{A}_{\partial}\right) \cup \Psi\left(\mathcal{A}_{\partial \Omega}\right)
$$

up to a set of $\mathcal{H}^{2 n-1}$-measure zero. 


\section{Fractional length}

In this section we define a fractional notion of length and show that in an appropriate limit as $\sigma$ goes to 1 , this converges to the $\mathcal{H}^{1}$ measure up to a multiplicative constant.

Let $\Omega$ be an open, bounded set with smooth boundary. Motivated by (19), given our curve $\mathcal{C}$, define the $\sigma$-length of $\mathcal{C}$ relative to $\Omega$ by

$$
\operatorname{Len}_{\sigma}(\mathcal{C}, \Omega):=\int_{\mathcal{D}(\mathcal{C})} r^{1-n-\sigma} \sup _{\mathbf{v} \in \mathcal{U}_{n} \cap\{\mathbf{u}\}^{\perp}} \chi_{\Omega}(p+r \mathbf{v}) d \mathcal{H}^{2 n}(p, \mathbf{u}, r),
$$

where $\mathcal{D}(\mathcal{C}):=\mathcal{D}_{\text {odd }}$. For simplicity, assume that $\mathcal{C}$ is contained in $\Omega$. Using the definitions in (36), the fractional length can be rewritten as

$$
\operatorname{Len}_{\sigma}(\mathcal{C}, \Omega)=\int_{\mathcal{D}_{\Omega}(\mathcal{C})} r^{1-n-\sigma} d \mathcal{H}^{2 n}(p, \mathbf{u}, r)
$$

To show that this definition yields a finite number, first notice that

$$
\mathcal{D}_{\Omega}(\mathcal{C}) \subseteq \Xi\left(\bigcup_{\xi \in \mathbb{R}^{+}} \mathcal{C} \times \mathcal{U}_{\perp}^{2} \times\{\xi\} \times[\xi, \xi+d(\Omega)]\right),
$$

where $d(\Omega)$ is the diameter of $\Omega$ and $\Xi$ is defined in (95) of the Appendix. To see this, consider $(p, \mathbf{u}, r) \in \mathcal{D}_{\Omega}(\mathcal{C})$. Since $D(p, \mathbf{u}, r)$ intersects $\mathcal{C}$ a finite number of times, we can find $z \in \mathcal{C} \cap D(p, \mathbf{u}, r)$ with minimum distance to $p$ such that $(p-z) \cdot \mathbf{u}=0$. Set $\xi=|p-z|$, $\mathbf{a}=(p-z) / \xi$, and $\mathbf{b}=\mathbf{u}$. It follows that $\Xi(z, \mathbf{a}, \mathbf{b}, \xi, r)=(p, \mathbf{u}, r)$. Since $z$ is the closest point on $\mathcal{C}$ to $p$ in $D(p, \mathbf{u}, r)$, we must have $\xi \leq r$ otherwise $D(p, \mathbf{u}, r)$ would not intersect $\mathcal{C}$. Moreover, $r \leq \xi+d(\Omega)$ since if this were not true then $\partial D(p, \mathbf{u}, r) \cap \Omega=\emptyset$. It follows that $(p, \mathbf{u}, r)$ is an element of the set on the right-hand side of (43), so (43) holds.

Thus, we can utilize the change of variables formula (96) in the Appendix to find that

$$
\begin{aligned}
\operatorname{Len}_{\sigma}(\mathcal{C}, \Omega) & \leq \int_{\mathcal{C}} \int_{0}^{\infty} \int_{\mathcal{U}_{\perp}^{2}} \int_{\xi}^{\xi+d(\Omega)} 2^{-1 / 2} r^{1-n-\sigma} \xi^{n-2}|\mathbf{b} \cdot \mathbf{t}(z)| d r d \mathcal{H}^{2 n-3}(\mathbf{a}, \mathbf{b}) d \xi d z \\
& \leq \mathcal{H}^{1}(\mathcal{C}) \mathcal{H}^{2 n-3}\left(\mathcal{U}_{\perp}^{2}\right) \int_{0}^{\infty} \int_{\xi}^{\xi+d(\Omega)} r^{1-n-\sigma} \xi^{n-2} d r d \xi \\
& =\frac{1}{2-n-\sigma} \mathcal{H}^{1}(\mathcal{C}) \mathcal{H}^{2 n-3}\left(\mathcal{U}_{\perp}^{2}\right) \int_{0}^{\infty}\left(\frac{\xi^{n-2}}{(\xi+d(\Omega))^{n-2+\sigma}}-\xi^{-\sigma}\right) d \xi
\end{aligned}
$$

and the remaining integral involving $\xi$ is finite.

The next goal is to show that the fractional length converges in an appropriate limit to the classical notion of length up to some multiplicative constant. Doing so will require the

following result. 
Lemma 3.1. For any $\mathbf{c} \in \mathcal{U}_{n}$, we have

$$
\int_{\mathcal{U}_{\perp}^{2}}|\mathbf{b} \cdot \mathbf{c}| d \mathcal{H}^{2 n-3}(\mathbf{a}, \mathbf{b})=\frac{4 \sqrt{2} \pi^{n-1}}{\Gamma\left(\frac{n+1}{2}\right) \Gamma\left(\frac{n-1}{2}\right)}
$$

where $\Gamma$ is the gamma function.

Proof. First notice that for any vector $\mathbf{v} \in \mathbb{R}^{n-1}$, by the area formula we have

$$
\int_{\mathcal{U}_{n-1}}|\mathbf{b} \cdot \mathbf{v}| d \mathbf{b}=2 \int_{\mathcal{U}_{n-2}} \int_{0}^{\pi / 2}|\mathbf{v}| \cos \theta(\sin \theta)^{n-3} d \theta d \mathcal{H}^{n-2}=\frac{\omega_{n-3} \Gamma\left(\frac{n-2}{2}\right)|\mathbf{v}|}{\Gamma\left(\frac{n}{2}\right)}=\frac{2 \pi^{\frac{n-2}{2}}|\mathbf{v}|}{\Gamma\left(\frac{n}{2}\right)}
$$

where

$$
\omega_{n-3}=\mathcal{H}^{n-3}\left(\mathcal{U}_{n-2}\right)=\frac{2 \pi^{\frac{n-2}{2}}}{\Gamma\left(\frac{n-2}{2}\right)} .
$$

Letting $P_{\mathbf{a}}$ denote the projection onto the plane orthogonal to a, we can compute using the coarea and area formulas that

$$
\begin{aligned}
\int_{\mathcal{U}_{\perp}^{2}}|\mathbf{b} \cdot \mathbf{c}| d \mathcal{H}^{2 n-3}(\mathbf{a}, \mathbf{b}) & =\int_{\mathcal{U}_{n}} \int_{\mathcal{U}_{n} \cap\{\mathbf{a}\}^{\perp}} \sqrt{2}\left|\mathbf{b} \cdot P_{\mathbf{a}} \mathbf{c}\right| d \mathbf{b} d \mathbf{a} \\
& =\frac{2 \sqrt{2} \pi^{\frac{n-2}{2}}}{\Gamma\left(\frac{n}{2}\right)} \int_{\mathcal{U}_{n}}\left|P_{\mathbf{a}} \mathbf{c}\right| d \mathbf{a} \\
& =\frac{4 \sqrt{2} \pi^{\frac{n-2}{2}}}{\Gamma\left(\frac{n}{2}\right)} \int_{\mathcal{U}_{n-1}} \int_{0}^{\pi / 2} \sin \theta(\sin \theta)^{n-2} d \theta d \mathcal{H}^{n-1} \\
& =\frac{4 \sqrt{2} \pi^{\frac{n-2}{2}}}{\Gamma\left(\frac{n}{2}\right)} \frac{\omega_{n-2} \Gamma\left(\frac{n}{2}\right) \Gamma\left(\frac{1}{2}\right)}{2 \Gamma\left(\frac{n+1}{2}\right)} \\
& =\frac{4 \sqrt{2} \pi^{n-1}}{\Gamma\left(\frac{n+1}{2}\right) \Gamma\left(\frac{n-1}{2}\right)}
\end{aligned}
$$

Theorem 3.2. If $\Omega \subseteq \mathbb{R}^{n}$ is an open, bounded set such that $\mathcal{C} \subseteq \Omega$, then

$$
\lim _{\sigma \uparrow 1}(1-\sigma) \operatorname{Len}_{\sigma}(\mathcal{C}, \Omega)=\frac{4 \pi^{n-1}}{\Gamma\left(\frac{n+1}{2}\right) \Gamma\left(\frac{n-1}{2}\right)(n-1)} \mathcal{H}^{1}(\mathcal{C}) .
$$

Proof. Begin by setting $\varepsilon:=(1-\sigma)^{1 / n}$ and

$$
\mathcal{D}_{\varepsilon}(\mathcal{C}):=\{(p, \mathbf{u}, r) \in \mathcal{D}(\mathcal{C}) \mid r \leq \varepsilon\} .
$$


One can show that

$$
\mathcal{D}_{\Omega}(\mathcal{C}) \backslash \mathcal{D}_{\varepsilon}(\mathcal{C}) \subseteq \Xi\left(\bigcup_{\xi \in \mathbb{R}^{+}} \mathcal{C} \times \mathcal{U}_{\perp}^{2} \times\{\xi\} \times[\max \{\xi, \varepsilon\}, \xi+d(\Omega)]\right),
$$

using an argument similar to that justifying (43). Thus, using the change of variables (96) there is a constant $C_{n}$ depending on $\mathcal{C}$ and $n$ such that

$$
\begin{aligned}
\int_{\mathcal{D}_{\Omega}(\mathcal{C}) \backslash \mathcal{D}_{\varepsilon}(\mathcal{C})} r^{1-n-\sigma} d \mathcal{H}^{2 n}(p, \mathbf{u}, r) \leq & C_{n} \int_{0}^{\infty} \int_{\max \{\xi, \varepsilon\}}^{\xi+d(\Omega)} \xi^{n-2} r^{1-n-\sigma} d r d \xi \\
= & \frac{C_{n}}{n+\sigma-2}\left[\int_{0}^{\varepsilon} \xi^{n-2}\left[\varepsilon^{2-n-\sigma}-(\xi+d(\Omega))^{n-2-\sigma}\right] d \xi\right. \\
& \left.+\int_{\varepsilon}^{\infty}\left[\xi^{-\sigma}-\xi^{n-2}(\xi+d(\Omega))^{2-n-\sigma}\right] d \xi\right] .
\end{aligned}
$$

Since

$$
\int_{0}^{\varepsilon} \xi^{n-2}\left[\varepsilon^{2-n-\sigma}-(\xi+d(\Omega))^{n-2-\sigma}\right] d \xi \leq \frac{\varepsilon^{1-\sigma}}{n-1}
$$

and

$$
\int_{\varepsilon}^{\infty}\left[\xi^{-\sigma}-\xi^{n-2}(\xi+d(\Omega))^{2-n-\sigma}\right] d \xi \leq \frac{d(\Omega)(n+\sigma-2) \varepsilon^{-\sigma}}{\sigma}
$$

it follows that

$$
\lim _{\sigma \uparrow 1}(1-\sigma) \int_{\mathcal{D}_{\Omega}(\mathcal{C}) \backslash \mathcal{D}_{\varepsilon}(\mathcal{C})} r^{1-n-\sigma} d \mathcal{H}^{2 n}(p, \mathbf{u}, r)=0
$$

Thus,

$$
\lim _{\sigma \uparrow 1}(1-\sigma) \operatorname{Len}_{\sigma}(\mathcal{C}, \Omega)=\lim _{\sigma \uparrow 1} \int_{\mathcal{D}_{\varepsilon}(\Omega)}(1-\sigma) r^{1-n-\sigma} d \mathcal{H}^{2 n}(p, \mathbf{u}, r) .
$$

Each $(p, \mathbf{u}, r) \in \mathcal{D}_{\varepsilon}(\mathcal{C})$ may intersect $\mathcal{C}$ multiple times, however we know it intersects $\mathcal{C}$ at least once. Thus, we can arbitrarily associate each $(p, \mathbf{u}, r)$ with some point $z \in \mathcal{C}$. Let $c(p, \mathbf{u}, r)$ denote the selected point in $\mathcal{C}$. We can think of $c$ as a mapping from $\mathcal{D}_{\varepsilon}(\mathcal{C})$ to $\mathcal{C}$. Many such mappings exist, but here we select one. For $z \in \mathcal{C}$ and $(\mathbf{a}, \mathbf{b}) \in \mathcal{U}_{\perp}^{2}$ set

$$
C_{\varepsilon}(z, \mathbf{a}, \mathbf{b}):=\left\{(\xi, r) \in \mathbb{R}^{+} \times \mathbb{R}^{+} \mid(z+\xi \mathbf{a}, \mathbf{b}, r) \in \mathcal{D}_{\varepsilon}(\mathcal{C}) \text { and } c(z+\xi \mathbf{a}, \mathbf{b}, r)=z\right\} .
$$

It follows from the definition of $c$ that the function $\Xi$ defined in (95) is injective on the set

$$
\bigcup_{(z, \mathbf{a}, \mathbf{b}) \in \mathcal{C} \times \mathcal{U}_{\perp}^{2}}\{z\} \times\{\mathbf{a}\} \times\{\mathbf{b}\} \times C_{\varepsilon}(z, \mathbf{a}, \mathbf{b}) .
$$

Thus, by the change of variables (96) we have

$$
\begin{aligned}
\int_{\mathcal{D}_{\varepsilon}(\Omega)} r^{1-n-\sigma} & d \mathcal{H}^{2 n}(p, \mathbf{u}, r) \\
& =\int_{\mathcal{C}} \int_{\mathcal{U}_{\perp}^{2}} \int_{C_{\varepsilon}(z, \mathbf{a}, \mathbf{b})} 2^{-1 / 2} r^{1-n-\sigma} \xi^{n-2}|\mathbf{b} \cdot \mathbf{t}(z)| d \mathcal{H}^{2}(\xi, r) d \mathcal{H}^{2 n-3}(\mathbf{a}, \mathbf{b}) d z
\end{aligned}
$$


Since $\mathcal{C}$ is a smooth curve, for all $(z, \mathbf{a}, \mathbf{b})$ such that $\mathbf{a}$ is not parallel to $\mathbf{t}(z)$ there is a $\varepsilon_{0}$ such that if $\varepsilon \leq \varepsilon_{0}$ we have

$$
C_{\varepsilon}(z, \mathbf{a}, \mathbf{b})=\left\{(\xi, r) \in \mathbb{R}^{+} \times \mathbb{R}^{+} \mid \xi \in[0, \varepsilon] \text { and } r \in[\xi, \varepsilon]\right\} .
$$

Thus,

$$
\begin{aligned}
\lim _{\sigma \uparrow 1}(1-\sigma) \int_{C_{\varepsilon}(z, \mathbf{a}, \mathbf{b})} r^{1-n-\sigma} \xi^{n-2} d \mathcal{H}^{2}(\xi, r) & =\lim _{\sigma \uparrow 1}(1-\sigma) \int_{0}^{\varepsilon} \int_{\xi}^{\varepsilon} r^{1-n-\sigma} \xi^{n-2} d r d \xi \\
& =\frac{1}{n-1} .
\end{aligned}
$$

Putting this together with (52) and (55) we find

$$
\lim _{\sigma \uparrow 1}(1-\sigma) \operatorname{Len}_{\sigma}(\mathcal{C}, \Omega)=\frac{2^{-1 / 2}}{n-1} \int_{\mathcal{C}} \int_{\mathcal{U}_{\perp}^{2}}|\mathbf{b} \cdot \mathbf{t}(z)| d \mathcal{H}^{2 n-3}(\mathbf{a}, \mathbf{b}) d z .
$$

With the help of Lemma 3.1, we obtain (47).

\section{Variation of $\operatorname{Len}_{\sigma}$ and nonlocal curvature}

This section is dedicated to computing the Euler-Lagrange equation associated with the functional $\operatorname{Len}_{\sigma}$. To compute this, we will use what is known as a transport theorem. The version of this transport theorem applicable here was established by Seguin [21]. For convenience, the statement of this theorem is formulated below in the context of the current setting.

Theorem 4.1. Let $\mathcal{I}$ be an open interval of $\mathbb{R}$ containing zero. For each $\epsilon \in \mathcal{I}$, let $\mathcal{O}_{\varepsilon}$ be an open subset of $\mathcal{D}$ that is locally of finite perimeter such that there exists a $(2 n-1)$ dimensional Riemannian manifold $\mathcal{N}$ and a function $\Theta \in C^{1}\left(\mathcal{I}, W^{1, \infty}\left(\mathcal{N}, \mathbb{R}^{n}\right)\right.$ ) such that the following conditions hold:

- for all $\varepsilon \in \mathcal{I}$, the differential of $\Theta_{\varepsilon}:=\Theta(\epsilon, \cdot): \mathcal{N} \rightarrow \mathbb{R}^{n}$ is injective, where it exists,

- $\partial^{*} \mathcal{O}_{\varepsilon}$ and $\Theta_{\varepsilon}(\mathcal{N})$ differ by a set of $\mathcal{H}^{2 n-1}$-measure zero, and

- $\mathcal{H}^{2 n-1}\left(\left\{(p, \mathbf{u}, r) \in \partial^{*} \mathcal{O}_{\varepsilon} \mid \mathcal{H}^{0}\left(\Theta_{\varepsilon}^{-1}(\{(p, \mathbf{u}, r)\})\right)>1\right\}\right)=0$.

It follows that for any $f \in W^{1,1}(\mathcal{D}, \mathbb{R})$ we have

$$
\left.\frac{d}{d \varepsilon} \int_{\mathcal{O}_{\varepsilon}} f d \mathcal{H}^{2 n}\right|_{\varepsilon=0}=\int_{\partial^{*} \mathcal{O}_{0}} f \mathbf{v} \cdot \mathbf{n} d \mathcal{H}^{2 n-1}
$$

where $\mathbf{n}$ is the exterior unit-normal to $\mathcal{O}_{0}$ and $\mathbf{v}$ is the "velocity" associated with $\Theta$ at $\varepsilon=0$, which is defined by

$$
\mathbf{v}(p, \mathbf{u}, r):=\left.\frac{\partial \Theta}{\partial \varepsilon}\left(\varepsilon, \Theta_{\varepsilon}^{-1}(p, \mathbf{u}, r)\right)\right|_{\varepsilon=0} \quad \text { for } \mathcal{H}^{2 n-1} \text {-a.e. }(p, \mathbf{u}, r) \in \partial^{*} \mathcal{O}_{\varepsilon}
$$


We can now compute the Euler-Lagrange equation associated with Len $_{\sigma}$.

Theorem 4.2. Let $\mathcal{C}$ be a $C^{1}$ curve in $\mathbb{R}^{n}$ whose closure is a manifold with boundary. A necessary and sufficient condition for the vanishing of the first variation with respect to curves with the same boundary as $\mathcal{C}$ of the $\sigma$-length relative to $\Omega$ is that for all $z \in \mathcal{C}$ we have

$$
\left(\int_{\mathcal{A}_{\text {odd }}^{+}(z)}-\int_{\mathcal{A}_{\text {even }}^{+}(z)}\right) \frac{(\mathbf{a} \cdot \mathbf{t}(z)) \mathbf{b}-(\mathbf{b} \cdot \mathbf{t}(z)) \mathbf{a}}{r^{1+\sigma}} d \mathcal{H}^{2 n-2}(\mathbf{a}, \mathbf{b}, r)=\mathbf{0},
$$

where

$$
\begin{aligned}
& \left.\mathcal{A}_{\text {odd }}^{+}(z):=\left\{(\mathbf{a}, \mathbf{b}, r) \in \mathcal{U}_{\perp}^{2} \times \mathbb{R}^{+} \mid \mathcal{H}^{0}(D(z+r \mathbf{a}, \mathbf{b}, r) \cap \mathcal{C}) \text { is odd }, \mathbf{b} \cdot \mathbf{t}(z)>0\right)\right\} \\
& \mathcal{A}_{\text {even }}^{+}(z):=\left\{(\mathbf{a}, \mathbf{b}, r) \in \mathcal{U}_{\perp}^{2} \times \mathbb{R}^{+} \mid \mathcal{H}^{0}(D(z+r \mathbf{a}, \mathbf{b}, r) \cap \mathcal{C}) \text { is even, } \mathbf{b} \cdot \mathbf{t}(z)>0\right\}
\end{aligned}
$$

Proof. Let $\mathbf{w}: \mathcal{C} \rightarrow \mathbb{R}^{n}$ be a $C^{1}$ function that satisfies two conditions: (i) $\mathbf{w}$ is zero at the endpoints of $\mathcal{C}$ and (ii) $\mathbf{w}(z)$ is orthogonal to the tangent space of $\mathcal{C}$ at $z \in \mathcal{C}$. For each $\varepsilon>0$ define the set

$$
\mathcal{C}_{\varepsilon}:=\{z+\varepsilon \mathbf{w}(z) \mid z \in \mathcal{C}\} .
$$

For sufficiently small $\varepsilon, \mathcal{C}_{\varepsilon}$ is a $C^{1}$ curve contained in $\Omega$. We seek a necessary and sufficient condition for

$$
\begin{aligned}
0 & =\lim _{\varepsilon \rightarrow 0} \frac{1}{\varepsilon}\left(\operatorname{Len}_{\sigma}\left(\mathcal{C}_{\varepsilon}, \Omega\right)-\operatorname{Len}_{\sigma}(\mathcal{C}, \Omega)\right) \\
& =\lim _{\varepsilon \rightarrow 0} \frac{1}{\varepsilon}\left(\int_{\mathcal{D}_{\Omega}\left(\mathcal{C}_{\varepsilon}\right)} r^{1-n-\sigma} d \mathcal{H}^{2 n}(p, \mathbf{u}, r)-\int_{\mathcal{D}_{\Omega}(\mathcal{C})} r^{1-n-\sigma} d \mathcal{H}^{2 n}(p, \mathbf{u}, r)\right) .
\end{aligned}
$$

To compute this limit, which is a derivative with respect to $\varepsilon$, we will use Theorem 4.1 To applying this result, we must show that the set $\mathcal{D}_{\Omega}\left(\mathcal{C}_{\varepsilon}\right)$, as it evolves with $\varepsilon$, has the properties listed in the theorem.

Towards this end, let $\mathcal{N}$ be the $(2 n-1)$-dimensional manifold defined as the disjoint union of $\mathcal{A}_{\partial}, \mathcal{A}_{\partial \Omega}$, and $\mathcal{A}_{\Omega \partial \mathcal{C}}$ (see (23), (37), and (39)), and define the function $\Theta: \mathcal{I} \times \mathcal{N} \rightarrow$ $\mathcal{D}$, where $\mathcal{I}$ is the interval of admissible $\varepsilon$ values being considered, by

$$
\Theta(\varepsilon, m):= \begin{cases}\Xi(m) & \text { if } m \in \mathcal{A}_{\Omega \partial \mathcal{C}}, \\ \Psi(z+\varepsilon \mathbf{w}(z), \mathbf{a}, \mathbf{b}, r) & \text { if } m=(z, \mathbf{a}, \mathbf{b}, r) \in \mathcal{A}_{\partial}, \\ \Psi(m) & \text { if } m \in \mathcal{A}_{\partial \Omega} .\end{cases}
$$

Notice that $\Theta$ is $C^{1}$ and that for all $\varepsilon, \Theta(\varepsilon, \mathcal{N})$ agrees with $\partial^{*} \mathcal{D}_{\Omega}\left(\mathcal{C}_{\varepsilon}\right)$ up to a set of $\mathcal{H}^{2 n-1}$-measure zero by the remarks at the end of Section 2 in particular (40). Setting $\Theta_{\varepsilon}:=\Theta(\varepsilon, \cdot)$, since for each $\varepsilon$ we have $\Theta_{\varepsilon}(\mathcal{N})=\partial^{*} \mathcal{D}_{\Omega}\left(\mathcal{C}_{\varepsilon}\right)$ up to a set of $\mathcal{H}^{2 n-1}$-measure zero, it follows from Items 5 and 6 of Lemma 2.1 and Proposition 2.4 that

$$
\mathcal{H}^{2 n-1}\left(\left\{(p, \mathbf{u}, r) \in \partial^{*} \mathcal{D}_{\Omega}\left(\mathcal{C}_{\varepsilon}\right) \mid \mathcal{H}^{0}\left(\Theta_{\varepsilon}^{-1}(\{(p, \mathbf{u}, r)\})>1\right)\right\}\right)=0
$$


These properties of $\Theta$ ensure that Theorem 4.1 can be applied. To do so, we need the velocity associated with $\Theta$. In this case, one can compute

$$
\mathbf{v}(p, \mathbf{u}, r)= \begin{cases}(\mathbf{w}(z), \mathbf{0}, 0) & \text { if }(p, \mathbf{u}, r) \in \mathcal{D}_{\partial 1}, \\ (\mathbf{0}, \mathbf{0}, 0) & \text { if }(p, \mathbf{u}, r) \in\left(\mathcal{D}_{\partial \mathcal{C}} \cap \mathcal{D}_{\Omega}\right) \cup \partial^{*} \mathcal{D}_{\Omega},\end{cases}
$$

where $z \in \mathcal{C}$ is the unique point in $D(p, \mathbf{u}, r) \cap \mathcal{C}$ when $(p, \mathbf{u}, r) \in \mathcal{D}_{\partial 1}$.

Using (58), the limit in (63) can be computed to obtain

$$
0=\int_{\partial^{*} \mathcal{D}(\mathcal{C})} r^{1-n-\sigma} \mathbf{v}(p, \mathbf{u}, r) \cdot \boldsymbol{\nu}(p, \mathbf{u}, r) d \mathcal{H}^{2 n-1}(p, \mathbf{u}, r)
$$

where $\boldsymbol{\nu}$ is the exterior unit normal to $\partial^{*} \mathcal{D}(\mathcal{C})$. The next step is to use Proposition 2.3 and the change variables formula (98) in the Appendix to find that the integral in the previous equation is equal to

$$
\int_{\mathcal{C}}\left(\int_{\mathcal{A}_{\text {odd }}(z)}-\int_{\mathcal{A}_{\text {even }}(z)}\right) \frac{\mathbf{w}(z) \cdot \operatorname{sgn}(\mathbf{b} \cdot \mathbf{t}(z))((\mathbf{a} \cdot \mathbf{t}(z)) \mathbf{b}-(\mathbf{b} \cdot \mathbf{t}(z)) \mathbf{a})}{\sqrt{2} r^{1+\sigma}} d \mathcal{H}^{2 n-2}(\mathbf{a}, \mathbf{b}, r) d z,
$$

where

$$
\begin{aligned}
& \mathcal{A}_{\text {odd }}(z):=\left\{(\mathbf{a}, \mathbf{b}, r) \in \mathcal{U}_{\perp}^{2} \times \mathbb{R}^{+} \mid \mathcal{H}^{0}(D(z+r \mathbf{a}, \mathbf{b}, r) \cap \mathcal{C}) \text { is odd }\right\} \\
& \mathcal{A}_{\text {even }}(z):=\left\{(\mathbf{a}, \mathbf{b}, r) \in \mathcal{U}_{\perp}^{2} \times \mathbb{R}^{+} \mid \mathcal{H}^{0}(D(z+r \mathbf{a}, \mathbf{b}, r) \cap \mathcal{C}) \text { is even }\right\}
\end{aligned}
$$

Notice that the domain of integration is symmetric under the transformation $\mathbf{b} \mapsto-\mathbf{b}$ and the integrand is an even function of $\mathbf{b}$. Thus, only integrating over those $\mathbf{b}$ such that $\mathbf{b} \cdot \mathbf{t}(z)>0$ and doubling the result we see that (68) can be written as

$$
\int_{\mathcal{C}}\left(\int_{\mathcal{A}_{\text {odd }}^{+}(z)}-\int_{\mathcal{A}_{\text {even }}^{+}(z)}\right) \frac{\mathbf{w}(z) \cdot 2(\mathbf{b} \cdot \mathbf{t}(z))((\mathbf{a} \cdot \mathbf{t}(z)) \mathbf{b}-(\mathbf{b} \cdot \mathbf{t}(z)) \mathbf{a})}{\sqrt{2} r^{1+\sigma}} d \mathcal{H}^{2 n-2}(\mathbf{a}, \mathbf{b}, r) d z .
$$

A standard argument then shows that being equal to zero holds for all normal variations $\mathbf{w}$ if and only if (60) holds.

Since a curve connecting two points of minimal length has zero curvature, the preceding result motivates that we define the nonlocal curvature-vector $\boldsymbol{\kappa}_{\sigma}$ at $z \in \mathcal{C}$ by

$$
\boldsymbol{\kappa}_{\sigma}(z):=\left(\int_{\mathcal{A}_{\text {even }}^{+}(z)}-\int_{\mathcal{A}_{\text {odd }}^{+}(z)}\right) \frac{(\mathbf{a} \cdot \mathbf{t}(z)) \mathbf{b}-(\mathbf{b} \cdot \mathbf{t}(z)) \mathbf{a}}{r^{1+\sigma}} d \mathcal{H}^{2 n-2}(\mathbf{a}, \mathbf{b}, r) .
$$

The integrals on the right-hand side must be computed in the principle value sense. Namely, first integrate over all discs with radius at least $\varepsilon>0$, and then take the limit as $\varepsilon$ goes to zero. Notice that this vector is orthogonal to the curve at $z$, however there is no reason 
to believe that this vector is parallel to the classical normal to the curve. The nonlocal scalar-curvature can be defined as the magnitude of this vector: $\kappa_{\sigma}(z):=\left|\boldsymbol{\kappa}_{\sigma}(z)\right|$.

A comparison of (72) with the nonlocal mean-curvature (15) when $n=2$ is in order. To do so, take the unit tangent of the curve $\mathbf{t}$ and rotate it $90^{\circ}$ clockwise to obtain a normal vector $\mathbf{n}$ to the curve. The mean curvature of a two-dimensional curve is obtained from the mean-curvature vector by dotting it with $\mathbf{n}$. Thus, consider

$\mathbf{n}(z) \cdot \boldsymbol{\kappa}_{\sigma}(z)=\left(\int_{\mathcal{A}_{\mathrm{even}}^{+}(z)}-\int_{\mathcal{A}_{\text {odd }}^{+}(z)}\right) \frac{(\mathbf{a} \cdot \mathbf{t}(z))(\mathbf{b} \cdot \mathbf{n}(z))-(\mathbf{b} \cdot \mathbf{t}(z))(\mathbf{a} \cdot \mathbf{n}(z))}{r^{1+\sigma}} d \mathcal{H}^{2 n-2}(\mathbf{a}, \mathbf{b}, r)$.

Focusing on the numerator of the integrand, notice that $\{\mathbf{t}(z), \mathbf{n}(z)\}$ and $\{\mathbf{a}, \mathbf{b}\}$ are both orthonormal basis for $\mathbb{R}^{2}$ and, hence, there is a orthogonal transformation $\mathbf{R}$ that takes $\{\mathbf{t}(z), \mathbf{n}(z)\}$ to $\{\mathbf{a}, \mathbf{b}\}$. It follows that

$$
(\mathbf{a} \cdot \mathbf{t}(z))(\mathbf{b} \cdot \mathbf{n}(z))-(\mathbf{b} \cdot \mathbf{t}(z))(\mathbf{a} \cdot \mathbf{n}(z))=\operatorname{det}(\mathbf{R}) .
$$

Thus, this value is either 1 or -1 , depending on if these two basis have the same or different orientation. Moreover, from the structure of orthogonal transformations in $\mathbb{R}^{2}$, we know that

$$
\operatorname{sgn} \operatorname{det}(\mathbf{R})=-\operatorname{sgn}((\mathbf{b} \cdot \mathbf{t}(z))(\mathbf{a} \cdot \mathbf{n}(z)),
$$

where $\operatorname{sgn} x=\frac{x}{|x|}$ is the sign function. Since in $\mathcal{A}_{\text {even }}^{+}(z)$ and $\mathcal{A}_{\text {odd }}^{+}(z)$ we always have $\mathbf{b} \cdot \mathbf{t}(z)>0$, this implies that $\operatorname{det}(\mathbf{R})=1$ if and only if $\mathbf{a} \cdot \mathbf{n}(z)<0$ and $\operatorname{det}(\mathbf{R})=-1$ if and only if $\mathbf{a} \cdot \mathbf{n}(z)>0$. Thus, if we define $\chi_{\mathcal{C}}: \mathcal{C} \times \mathcal{U}^{2} \times \mathbb{R}^{+} \rightarrow \mathbb{R}$ by

$$
\chi_{C}(z, \mathbf{a}, \mathbf{b}, r):=\left\{\begin{array}{cc}
1 & \left((\mathbf{a}, \mathbf{b}, r) \in \mathcal{A}_{\text {even }}^{+}(z) \text { and } \mathbf{a} \cdot \mathbf{n}(z)<0\right) \\
& \text { or }\left((\mathbf{a}, \mathbf{b}, r) \in \mathcal{A}_{\text {odd }}^{+}(z) \text { and } \mathbf{a} \cdot \mathbf{n}(z)>0\right) \\
-1 & \left((\mathbf{a}, \mathbf{b}, r) \in \mathcal{A}_{\text {even }}^{+}(z) \text { and } \mathbf{a} \cdot \mathbf{n}(z)>0\right) \\
& \text { or }\left((\mathbf{a}, \mathbf{b}, r) \in \mathcal{A}_{\text {odd }}^{+}(z) \text { and } \mathbf{a} \cdot \mathbf{n}(z)<0\right) \\
0 & \text { otherwise },
\end{array}\right.
$$

then (73) can be written as

$$
\mathbf{n}(z) \cdot \boldsymbol{\kappa}_{\sigma}(z)=\int_{\left(\mathcal{U}_{\perp}^{2} \times \mathbb{R}^{+}\right)^{+}(z)} \frac{\chi_{\mathcal{C}}(\mathbf{a}, \mathbf{b}, r)}{r^{1+\sigma}} d \mathcal{H}^{2 n-2}(\mathbf{a}, \mathbf{b}, r),
$$

where

$$
\left(\mathcal{U}_{\perp}^{2} \times \mathbb{R}^{+}\right)^{+}(z):=\left\{(\mathbf{a}, \mathbf{b}, r) \in \mathcal{U}_{\perp}^{2} \times \mathbb{R}^{+} \mid \mathbf{b} \cdot \mathbf{t}(z)>0\right\} .
$$

Using the change of variables $(\mathbf{a}, \mathbf{b}, r) \mapsto z+2 r \mathbf{a}$ shows that $\mathbf{n}(z) \cdot \boldsymbol{\kappa}_{\sigma}(z)$ agrees with $H_{\sigma}(z)$ in (15) with $n=2$ up to a multiplicative constant. It is not surprising that they differ by such a constant since $H_{\sigma}(z)$ in (15) is normalized to ensure that it converges in the appropriate limit to the classical mean curvature, but $\boldsymbol{\kappa}_{\sigma}(z)$ has not been properly normalized. The investigation of what this normalization constant should be is left to future work. 


\section{Acknowledgments}

I would like to thank Cornelia Mihaila for spotting an error in the published version of this manuscript and for comments on this corrected version.

\section{Appendix: some change of variables}

Let $\mathcal{C}$ be a $C^{1}$ curve in $\mathbb{R}^{n}$.

Lemma 4.3. Consider the function

$$
\Phi: \mathcal{C} \times \mathcal{U}_{\perp}^{2} \times \mathbb{R}^{+} \rightarrow \mathbb{R}^{n} \times \mathbb{R}^{n}
$$

defined by

$$
\Phi(z, \mathbf{a}, \mathbf{b}, \xi):=(z+\xi \mathbf{a}, \mathbf{b}) \quad \text { for } \operatorname{all}(z, \mathbf{a}, \mathbf{b}, \xi) \in \mathcal{C} \times \mathcal{U}_{\perp}^{2} \times \mathbb{R}^{+}
$$

If $\mathcal{A}$ is a subset of $\mathcal{C} \times \mathcal{U}_{\perp}^{2} \times \mathbb{R}^{+}$and $f: \Phi(\mathcal{A}) \rightarrow \mathbb{R}$ is an integrable function, then

$$
\begin{aligned}
& \int_{\Phi(\mathcal{A})}\left[\sum_{(z, \mathbf{a}, \mathbf{b}, \xi) \in \Phi^{-1}(p, \mathbf{u})} f(p, \mathbf{u})\right] d \mathcal{H}^{2 n-1}(p, \mathbf{u}) \\
&=\int_{\mathcal{A}} f(z+\xi \mathbf{a}, \mathbf{b}) 2^{-1 / 2} \xi^{n-2}|\mathbf{b} \cdot \mathbf{t}(z)| d \mathcal{H}^{2 n-1}(z, \mathbf{a}, \mathbf{b}, \xi)
\end{aligned}
$$

where $\mathbf{t}(z)$ is a unit-vector tangent to the curve $\mathcal{C}$ at the point $z$.

Proof. It suffices to prove the result for a set of the form $\mathcal{A}=\mathcal{C}_{\mathcal{A}} \times \mathcal{U}_{\mathcal{A}} \times \mathcal{I}_{\mathcal{A}}$, where $\mathcal{C}_{\mathcal{A}} \subseteq \mathcal{C}$, $\mathcal{U}_{\mathcal{A}} \subseteq \mathcal{U}_{\perp}^{2}$, and $\mathcal{I}_{\mathcal{A}} \subseteq \mathbb{R}^{+}$. Moreover, by employing a partition of unity, we can reduce the problem to the case where $\mathcal{U}_{\mathcal{A}}$ is covered by one chart. Thus, there is a set $U_{\mathcal{A}} \subseteq \mathbb{R}^{2 n-3}$ and a diffeomorphism $\chi: U_{\mathcal{A}} \rightarrow \mathcal{U}_{\mathcal{A}}$. Since $\mathcal{U}_{\mathcal{A}} \subseteq \mathbb{R}^{n} \times \mathbb{R}^{n}$, we can view this function as $\chi=\left(\chi_{1}, \chi_{2}\right)$, where $\boldsymbol{\chi}_{1}, \chi_{2}: \mathbb{R}^{2 n-3} \rightarrow \mathbb{R}^{n}$. Recall that if $g$ is an integrable function defined on $\mathcal{U}_{\mathcal{A}}$, then

$$
\int_{\mathcal{U}_{\mathcal{A}}} g(\mathbf{a}, \mathbf{b}) d \mathcal{H}^{2 n-3}(\mathbf{a}, \mathbf{b})=\int_{U_{\mathcal{A}}} g\left(\chi_{1}(w), \boldsymbol{\chi}_{2}(w)\right) J_{\chi}(w) d w
$$

where $J_{\chi}=\sqrt{\operatorname{det}\left(\nabla \chi^{\top} \nabla \boldsymbol{\chi}\right)}$ is the Jacobian of $\boldsymbol{\chi}$. Also, if $h$ is an integrable function defined on $\mathcal{C}_{\mathcal{A}}$ and $\phi$ is a parameterization of $\mathcal{C}$, then

$$
\int_{\mathcal{C}_{\mathcal{A}}} h(z) d z=\int_{C_{\mathcal{A}}} h(\phi(s))\left|\phi^{\prime}(s)\right| d s
$$

where $C_{\mathcal{A}}$ is the subset of $\mathbb{R}$ such that $\phi\left(C_{\mathcal{A}}\right)=\mathcal{C}_{\mathcal{A}}$. 
Set $A:=C_{\mathcal{A}} \times U_{\mathcal{A}} \times \mathcal{I}_{\mathcal{A}}$ and define $\tilde{\Phi}: A \rightarrow \Phi(\mathcal{A})$ by

$$
\tilde{\Phi}(s, w, \xi):=\Phi\left(\phi(s), \chi_{1}(w), \chi_{2}(w), \xi\right)=\left(\phi(s)+\xi \chi_{1}(w), \chi_{2}(w)\right)
$$

and $\tilde{f}: A \rightarrow \mathbb{R}$ by

$$
\tilde{f}(s, w, \xi)=f\left(\phi(s)+\xi \chi_{1}(w), \chi_{2}(w)\right) .
$$

By the Area Formula (see Theorem 2.71 of Ambrosio, Fusco, and Pallara [2]), it follows that

$$
\int_{\tilde{\Phi}(A)}\left[\sum_{(s, w, \xi) \in \Phi^{-1}(p, \mathbf{u})} \tilde{f}(s, w, \xi)\right] d \mathcal{H}^{2 n-1}(p, \mathbf{u})=\int_{A} \tilde{f}(s, w, \xi) J_{\tilde{\Phi}}(s, w, \xi) d \mathcal{H}^{2 n-1}(s, w, \xi) .
$$

Thus, by (811) and (82), the result will follow once it is shown that

$$
J_{\tilde{\Phi}}=2^{-1 / 2} \xi^{n-2}\left|\chi_{2} \cdot \mathbf{t}\right|\left|\phi^{\prime}\right| J_{\chi} .
$$

To establish this, first notice that

$$
\nabla \tilde{\Phi}=\left(\begin{array}{c|c|c}
1 & 2 n-3 & 1 \\
\phi^{\prime} & \xi \nabla \chi_{1} & \boldsymbol{\chi}_{1} \\
\hline \mathbf{0} & \nabla \chi_{2} & \mathbf{0}
\end{array}\right) \begin{aligned}
& n \\
& n
\end{aligned}
$$

and hence, noting that $\left|\boldsymbol{\chi}_{1}\right|^{2}=1$ and $\nabla \boldsymbol{\chi}_{1}^{\top} \boldsymbol{\chi}_{1}=\mathbf{0}$, we have

$$
\nabla \tilde{\Phi}^{\top} \nabla \tilde{\Phi}=\left(\begin{array}{c|c|c}
\left|\phi^{\prime}\right|^{2} & \xi \phi^{\prime \top} \nabla \chi_{1} & \chi_{1} \cdot \phi^{\prime} \\
\hline \xi \nabla \chi_{1}^{\top} \phi^{\prime} & \xi^{2} \nabla \chi_{1}^{\top} \nabla \chi_{1}+\nabla \chi_{2}^{\top} \nabla \chi_{2} & 0 \\
\hline \chi_{1} \cdot \phi^{\prime} & 0 & 1
\end{array}\right) .
$$

Since switching rows or columns of a matrix does not change the absolute value of its determinant, we have

$$
\left|\operatorname{det}\left(\nabla \tilde{\Phi}^{\top} \nabla \tilde{\Phi}\right)\right|=\left|\operatorname{det}\left(\begin{array}{c|c|c}
\left|\phi^{\prime}\right|^{2} & \chi_{1} \cdot \phi^{\prime} & \xi \phi^{\prime \top} \nabla \boldsymbol{\chi}_{1} \\
\hline \boldsymbol{\chi}_{1} \cdot \phi^{\prime} & 1 & 0 \\
\hline \xi \nabla \boldsymbol{\chi}_{1}^{\top} \phi^{\prime} & 0 & \xi^{2} \nabla \boldsymbol{\chi}_{1}^{\top} \nabla \boldsymbol{\chi}_{1}+\nabla \boldsymbol{\chi}_{2}^{\top} \nabla \boldsymbol{\chi}_{2}
\end{array}\right)\right| .
$$

Recall that the determinant of a block matrix can be computed using

$$
\operatorname{det}\left(\begin{array}{l|l}
\mathbf{A} & \mathbf{B} \\
\hline \mathbf{C} & \mathbf{D}
\end{array}\right)=\operatorname{det}(\mathbf{A}) \operatorname{det}\left(\mathbf{D}-\mathbf{C A}^{-1} \mathbf{B}\right)
$$


This identity will be used with $\mathbf{D}=\xi^{2} \nabla \boldsymbol{\chi}_{1}^{\top} \nabla \boldsymbol{\chi}_{1}+\nabla \boldsymbol{\chi}_{2}^{\top} \nabla \boldsymbol{\chi}_{2}$. Let $P_{\boldsymbol{\chi}_{1}}$ denote the projection onto the plane orthogonal to the vector $\chi_{1}$. After some computation, using the identity $\left|P_{\chi_{1}} \phi^{\prime}\right|^{2}=\left|\phi^{\prime}\right|^{2}-\left(\chi_{1} \cdot \phi^{\prime}\right)^{2}$, one finds that

$$
\left|\operatorname{det}\left(\nabla \tilde{\Phi}^{\top} \nabla \tilde{\Phi}\right)\right|=\left|P_{\boldsymbol{\chi}_{1}} \phi^{\prime}\right|^{2}\left|\operatorname{det}\left[\nabla \boldsymbol{\chi}_{1}^{\top}\left(\xi^{2} \mathbf{1}_{n}-\frac{\xi^{2}}{\left|P_{\boldsymbol{\chi}_{1}} \phi^{\prime}\right|^{2}} P_{\boldsymbol{\chi}_{1}} \phi^{\prime} \otimes P_{\boldsymbol{\chi}_{1}} \phi^{\prime}\right) \nabla \boldsymbol{\chi}_{1}+\nabla \boldsymbol{\chi}_{2}^{\top} \nabla \boldsymbol{\chi}_{2}\right]\right| \text {. }
$$

where $\mathbf{1}_{n}$ is the identity function on $\mathbb{R}^{n}$. It follows that

$$
\left|\operatorname{det}\left(\nabla \tilde{\Phi}^{\top} \nabla \tilde{\Phi}\right)\right|=\left|P_{\boldsymbol{\chi}_{1}} \phi^{\prime}\right|^{2}\left|\operatorname{det}\left[\nabla \boldsymbol{\chi}^{\top}\left(\begin{array}{c|c}
\xi^{2} \mathbf{1}_{n}-\xi^{2} \tilde{\mathbf{t}} \otimes \tilde{\mathbf{t}} & \mathbf{0} \\
\hline \mathbf{0} & \mathbf{1}_{n}
\end{array}\right) \nabla \boldsymbol{\chi}\right]\right|
$$

where $\tilde{\mathbf{t}}=P_{\boldsymbol{\chi}_{1}} \phi^{\prime} /\left|P_{\boldsymbol{\chi}_{1}} \phi^{\prime}\right|$. To simplify the right-hand side of the previous equation, set $\mathbf{L}$ equal to the square $2 n \times 2 n$ matrix in the previous equation between $\nabla \chi^{\top}$ and $\nabla \boldsymbol{\chi}$ and recall the fact that

$$
\operatorname{det}\left(\nabla \chi^{\top} \mathbf{L} \nabla \boldsymbol{\chi}\right)=J_{\chi}^{2} \operatorname{det}\left(\mathbf{I}^{\top} \mathbf{L I}\right)
$$

where $\mathbf{I}$ is the natural injection of the range of $\nabla \boldsymbol{\chi}$ into $\mathbb{R}^{2 n}$. One can find an orthonormal basis for $\mathbb{R}^{n}$ of the form

$$
\left(\mathbf{e}_{1}, \mathbf{e}_{2}, \ldots, \mathbf{e}_{n-2}, \chi_{1}, \chi_{2}\right)
$$

such that $\tilde{\mathbf{t}}$ can be written as a linear combination of $\mathbf{e}_{n-2}$ and $\boldsymbol{\chi}_{2}$. Notice that

$$
\left\{\left(\mathbf{e}_{1}, \mathbf{0}\right), \ldots,\left(\mathbf{e}_{n-2}, \mathbf{0}\right),\left(\mathbf{0}, \mathbf{e}_{1}\right), \ldots,\left(\mathbf{0}, \mathbf{e}_{n-2}\right), \frac{1}{\sqrt{2}}\left(\chi_{2},-\chi_{1}\right)\right\}
$$

is an orthonormal basis for the tangent space of $\mathcal{U}_{\perp}^{2}$ at $\left(\boldsymbol{\chi}_{1}, \boldsymbol{\chi}_{2}\right)$. By writing the matrix

$$
\mathbf{I}^{\top}\left(\begin{array}{c|c}
\xi^{2} \mathbf{1}_{n}-\xi^{2} \tilde{\mathbf{t}} \otimes \tilde{\mathbf{t}} & \mathbf{0} \\
\hline \mathbf{0} & \mathbf{1}_{n}
\end{array}\right) \mathbf{I}
$$

relative to the basis (93), one can compute its determinant and, hence, putting together (91) and (92), we have that

$$
\left|\operatorname{det}\left(\nabla \boldsymbol{\chi}^{\top} \mathbf{L} \nabla \boldsymbol{\chi}\right)\right|=2^{-1 / 2} \xi^{2 n-4}\left|P_{\boldsymbol{\chi}_{1}} \phi^{\prime}\right|^{2}\left|\chi_{2} \cdot \tilde{\mathbf{t}}\right|^{2} J_{\chi}^{2}
$$

Since $\left|P_{\boldsymbol{\chi}_{1}} \phi^{\prime}\right|^{2}\left|\chi_{2} \cdot \tilde{\mathbf{t}}\right|^{2}=\left|\chi_{2} \cdot P_{\chi_{1}} \mathbf{t}\right|^{2}\left|\phi^{\prime}\right|^{2}=\left|\chi_{2} \cdot \mathbf{t}\right|^{2}\left|\phi^{\prime}\right|^{2}$, this proves (86).

A slight variation on the change of variables formula (80) will be needed. Namely, we will require a change of variables associated with the function

$$
\Xi: \mathcal{C} \times \mathcal{U}_{\perp}^{2} \times \mathbb{R}^{+} \times \mathbb{R}^{+} \rightarrow \mathbb{R}^{n} \times \mathbb{R}^{n} \times \mathbb{R}
$$



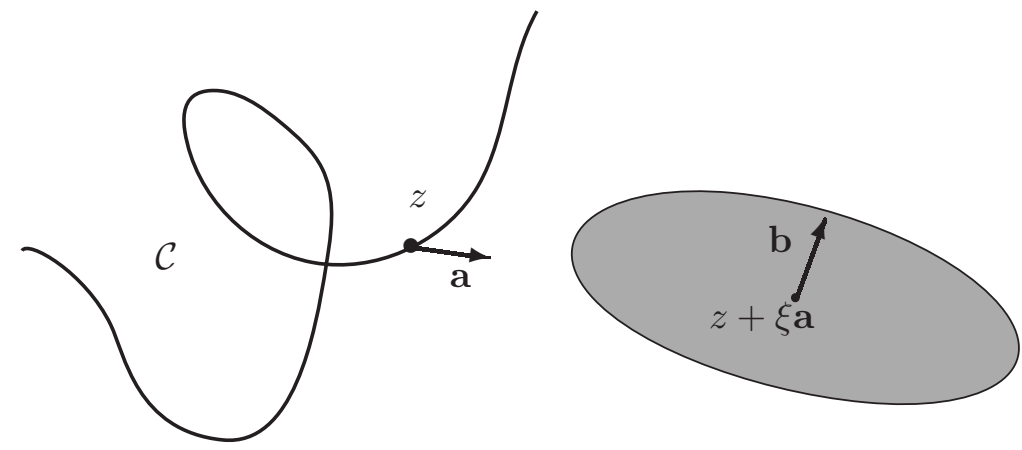

Figure 2: A depiction of the disc associated with $\Xi(z, \mathbf{a}, \mathbf{b}, \xi, r)$.

defined by

$$
\Xi(z, \mathbf{a}, \mathbf{b}, \xi, r):=(z+\xi \mathbf{a}, \mathbf{b}, r) \quad \text { for all }(z, \mathbf{a}, \mathbf{b}, \xi, r) \in \mathcal{C} \times \mathcal{U}_{\perp}^{2} \times \mathbb{R}^{+} \times \mathbb{R}^{+} .
$$

The function $\Xi$ allows us to describe discs using points on the curve $\mathcal{C}$; see Figure 2 Since $\Xi$ acts like the identity on the last variable $r$, the previous lemma immediately implies that if $\mathcal{A}$ is a subset of $\mathcal{C} \times \mathcal{U}_{\perp}^{2} \times \mathbb{R}^{+} \times \mathbb{R}^{+}$and $f: \Xi(\mathcal{A}) \rightarrow \mathbb{R}$ is an integrable function, then

$$
\begin{aligned}
\int_{\Xi(\mathcal{A})}\left[\sum_{(z, \mathbf{a}, \mathbf{b}, \xi, r) \in \Xi^{-1}(p, \mathbf{u}, r)}\right. & f(p, \mathbf{u}, r)] d \mathcal{H}^{2 n}(p, \mathbf{u}, r) \\
= & \int_{\mathcal{A}} f(z+\xi \mathbf{a}, \mathbf{b}, r) 2^{-1 / 2} \xi^{n-2}|\mathbf{b} \cdot \mathbf{t}(z)| d \mathcal{H}^{2 n}(z, \mathbf{a}, \mathbf{b}, \xi, r) .
\end{aligned}
$$

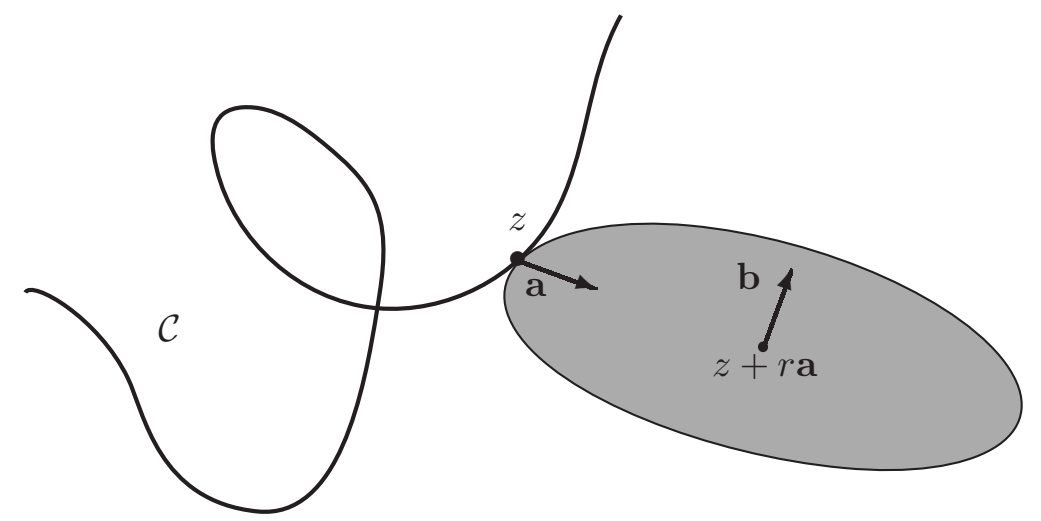

Figure 3: A depiction of the disc associated with $\Psi(z, \mathbf{a}, \mathbf{b}, r)$.

Another useful change of variables will be needed. This time the function, which is closely related to $\Xi$, will allow us to describe all discs whose boundary touches the curve $\mathcal{C}$; 
see Figure 3, Define the function

$$
\Psi: \mathcal{C} \times \mathcal{U}_{\perp}^{2} \times \mathbb{R}^{+} \rightarrow \mathbb{R}^{n} \times \mathbb{R}^{n} \times \mathbb{R}
$$

by

$$
\Psi(z, \mathbf{a}, \mathbf{b}, r):=(z+r \mathbf{a}, \mathbf{b}, r) \quad \text { for all }(z, \mathbf{a}, \mathbf{b}, r) \in \mathcal{C} \times \mathcal{U}_{\perp}^{2} \times \mathbb{R}^{+} .
$$

It is possible to show that if $\mathcal{A}$ is a subset of $\mathcal{C} \times \mathcal{U}_{\perp}^{2} \times \mathbb{R}^{+}$and $f: \Psi(\mathcal{A}) \rightarrow \mathbb{R}$ is an integrable function, then

$$
\begin{aligned}
& \int_{\Psi(\mathcal{A})}\left[\sum_{(z, \mathbf{a}, \mathbf{b}, r) \in \Psi^{-1}(p, \mathbf{u}, r)} f(p, \mathbf{u}, r)\right] d \mathcal{H}^{2 n}(p, \mathbf{u}, r) \\
& =\int_{\mathcal{A}} f(z+r \mathbf{a}, \mathbf{b}, r) 2^{-1 / 2} r^{n-2} \sqrt{\left(1+r^{2}\right)(\mathbf{a} \cdot \mathbf{t}(z))^{2}+2(\mathbf{b} \cdot \mathbf{t}(z))^{2}} d \mathcal{H}^{2 n}(z, \mathbf{a}, \mathbf{b}, r) .
\end{aligned}
$$

The proof of this result is similar to that of Lemma 4.3 and, thus, will not be presented.

\section{References}

[1] Abatangelo, A., and Valdinoci, E. A notion of nonlocal curvature. Numerical Functional Analysis and Optimization 35 (2014), 793-815.

[2] Ambrosio, L., Fusco, N., and Pallara, D. Functions of Bounded Variation and Free Discontinuity Problems. Oxford Science Publications, Oxford, 2000.

[3] Ambrosio, L., Philippis, G. D., and Martinazzi, L. Gamma-convergence of nonlocal perimeter functionals. Manuscripta Mathematica 134 (2011), 377-403.

[4] Caffarelli, L., Roquejoffre, J. M., and Savin, O. Nonlocal minimal surfaces. Communications on Pure and Applied Mathematics 63 (2010), 1111-1144.

[5] Caffarelli, L., and Valdinoci, E. Uniform estimates and limiting arguments for nonlocal minimal surfaces. Calculus of Variations and Partial Differential Equations 41 (2011), 203-240.

[6] Caffarelli, L., and Valdinoci, E. Regularity properties of nonlocal minimal surfaces via limiting arguments. Advances in Mathematics 248 (2013), 843-871.

[7] Chambolle, A., Marini, M., and Ponsiglione, M. Nonlocal curvature flows. Archive for Rational Mechanics and Analysis 218 (2015), 1263-1329.

[8] Chambolle, A., Morini, M., and Ponsiglione, M. A nonlocal mean curvature flow and its semi-implicit time-discrete approximation. SIAM Journal of Mathematical Analysis 44 (2012), 4048-4077. 
[9] Chambolle, A., Morini, M., and Ponsiglione, M. Minimizing movements and level set approaches to nonlocal variational geometric flows. In Geometric Partial Differential Equations proceedings (Pisa, 2013), A. Chambolle, M. Novaga, and E. Valdinoci, Eds., Scuola Normale Superiore, pp. 93-104.

[10] Dipierro, S., Figalli, A., Palatucci, G., and Valdinoci, E. Asymptotics of the $s$ perimeter as $s \downarrow 0$. Discrete and Continuous Dynamical Systems 33 (2013), 2777-2790.

[11] Dipierro, S., Savin, O., and Valdinoci, E. Boundary behaviour of nonlocal minimal surfaces. Journal of Functional Analysis 272 (2017), 1791-1851.

[12] Dipierro, S., and Valdinoci, E. Nonlocal minimal surfaces: interior regularity, quantitative estimates and boundary stickiness. In Recent developments in nonlocal theory, G. Palatucci and T. Kuusi, Eds. De Gruyter, Berlin, 2018, ch. 4, pp. 165-209.

[13] Dipierro, S., and Valdinoci, E. Nonlocal minimal surfaces: Interior regularity, quantitative estimates and boundary stickiness. In Recent developments in nonlocal theory. De Gruyter, Berlin, 2018, pp. 165-209.

[14] Federer, H. Geometric Measure Theory. Springer-Verlag, Berlin Heidelberg New York, 1969.

[15] Figalli, A., and Valdinoci, E. Regularity and bernstein-type results for nonlocal minimal surfaces. Journal für die reine und angewandte Mathematik (published on line 2015-04-28).

[16] Giusti, E. Minimal surfaces and functions of bounded variation. Birkhüser Verlag, Basel, 1984.

[17] Imbert, C. Level set approach for fractional mean curvature flows. Interfaces Free Boundaries 11 (2009), 153-176.

[18] Paroni, R., Podio-Guidugli, P., and Seguin, B. On the nonlocal curvatures of open surfaces. Communications on Pure and Applied Analysis 17 (2018), 709-727.

[19] Savin, O., and Valdinoci, E. Regularity of nonlocal minimal cones in dimension 2. Calculus of Variations and Partial Differential Equations 48 (2013), 33-39.

[20] Seguin, B. A fractional notion of length and an associated nonlocal curvature. The Journal of Geometric Analysis 30 (2020), 161-181.

[21] Seguin, B. A transport theorem for nonconvecting open sets on an embedded manifold. Continuum Mechanics and Thermodynamics 32 (2020), 1-8.

[22] Visintin, A. Generalized coarea formula and fractal sets. Japan Journal of Industrial and Applied Mathematics 8 (1991), 175-201. 